SUPPORTING INFORMATION

FOR

DETERMINATION OF MOLECULAR STRUCTURE USING

VIBRATIONAL CIRCULAR

DICHROISM SPECTROSCOPY: THE KETO-LACTONE

PRODUCT OF BAEYER-VILLIGER

OXIDATION OF (+)-(1R,5S)-BICYCLO[3.3.1]NONANE-2,7-DIONE

\author{
P. J. Stephens*, D. M. McCann, F. J. Devlin and T. C. Flood \\ Department of Chemistry, University of Southern California \\ Los Angeles, CA 90089-0482 \\ E. Butkus and S. Stončius \\ Department of Organic Chemistry, Vilnius University
}

Naugarduko 24, LT-03225 Vilnius, Lithuania

and

J. R. Cheeseman

Gaussian Inc., 340 Quinnipiac St, Bidg 40

Wallingford, CT 06492-4050 
Contents:

Figures 1-4: B3PW91/TZ2P Structures of 2a-2d.

Table 1: Dihedral angles of the CC conformations of 2a-2d at the B3LYP/6-31G*, B3LYP/TZ2P and B3PW91/TZ2P levels.

Figure 5: Experimental IR (a) and VCD (b) spectra of a $0.061 \mathrm{M}$ solution of (+)-2 in $\mathrm{CHCl}_{3}$.

Table 2 Calculated Frequencies, Dipole Strengths and Rotational Strengths for the CC Conformation of (1R,6R)-2a and Experimental Frequencies, Dipole Strengths and Rotational Strengths for $(+)-2$.

Table 3 Calculated Frequencies, Dipole Strengths and Rotational Strengths for the CC Conformation of (1R,6S)-2b.

Table 4 Calculated Frequencies, Dipole Strengths and Rotational Strengths for the CC Conformation of (1R,6S)-2c.

Table 5 Calculated Frequencies, Dipole Strengths and Rotational Strengths for the CC Conformation of (1S,6R)-2d.

Figure 6: Comparison of the B3LYP/TZ2P IR Spectra of 2a-2d to the Experimental IR Spectrum of $(+)-2$.

Figure 7: Comparison of the B3LYP/TZ2P VCD Spectra of 2a-2d to the Experimental VCD Spectrum (+)-2.

Figure 8: Comparison of the B3PW91/TZ2P and B3LYP/TZ2P IR spectra of 2a to the experimental IR spectrum of $(+)-2$.

Figure 9: Comparison of the B3PW91/TZ2P and B3LYP/TZ2P VCD spectra of $(1 \mathrm{R}, 6 \mathrm{R})-2 \mathrm{a}$ to the experimental VCD spectrum of $(+)-2$. 
Figure 10: Comparison of B3PW91/TZ2P frequencies, dipole strengths and rotational strengths for (1R,6R)-2a to the experimental parameters for $(+)-2$.

Figure 11: Comparison of B3PW91/TZ2P and B3LYP/TZ2P frequencies, dipole strengths and rotational strengths for $(1 \mathrm{R}, 6 \mathrm{R})-\mathbf{2 a}$ to the experimental parameters for $(+)-$

2.

Table 6: Observed chemical shifts and coupling constants of 2.

Figure 12: Calculated ${ }^{1} \mathrm{H}$ NMR spectrum of the CC conformation of $\mathbf{2 b} \quad$ S20

Figure 13: Calculated ${ }^{1} \mathrm{H}$ NMR spectrum of the CC conformation of 2c. $\quad$ S21

Figure 14: Calculated ${ }^{1} \mathrm{H}$ NMR spectrum of the CC conformation of $\mathbf{2 d} \quad$ S22

Table 7: Calculated ${ }^{1} \mathrm{H}$ chemical shifts and coupling constants for the $\mathrm{CC}$ conformation of 2 a.

Figure 15: Calculated ${ }^{13} \mathrm{C}$ NMR chemical shifts of 2a-2d compared with the experimental ${ }^{13} \mathrm{C}$ chemical shifts of 2 .

Table 8: Calculated Specific Rotations, Excitation Energies and Rotational Strengths

for $\mathbf{2 b}-\mathbf{2 d}$.

Table 9: Variation of Calculated Specific Rotations and Electronic Excitation Energies and Rotational Strengths of the CC conformation of (1R,6R)-2a with Functional, Basis Set and Geometry. S25-

Cartesian Coordinate B3LYP/6-31G*, B3LYP/TZ2P and B3PW91/TZ2P

Geometries of 2a-2d. 


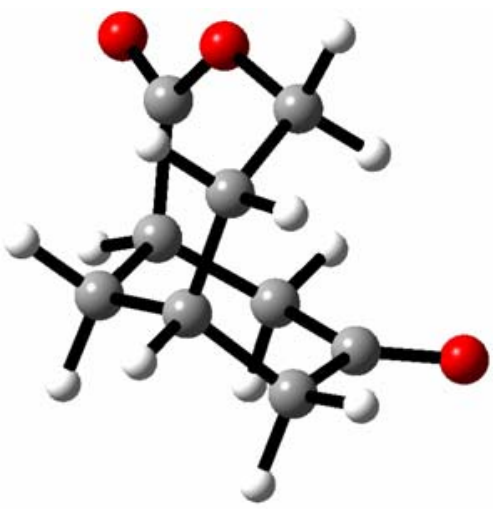

Figure 1: B3PW91/TZ2P Structure of the CC conformation of (1R,6R)-2a.

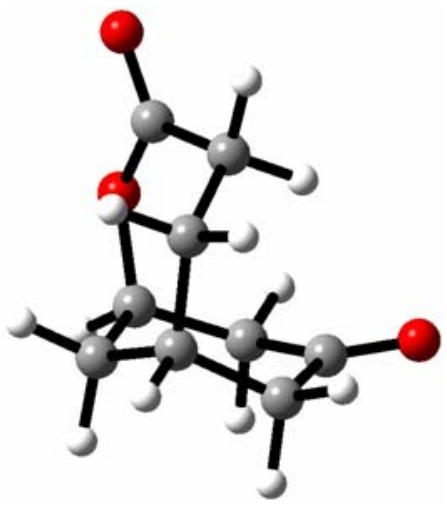

Figure 2: B3PW91/TZ2P Structure of the CC conformation of (1R,6S)-2b. 


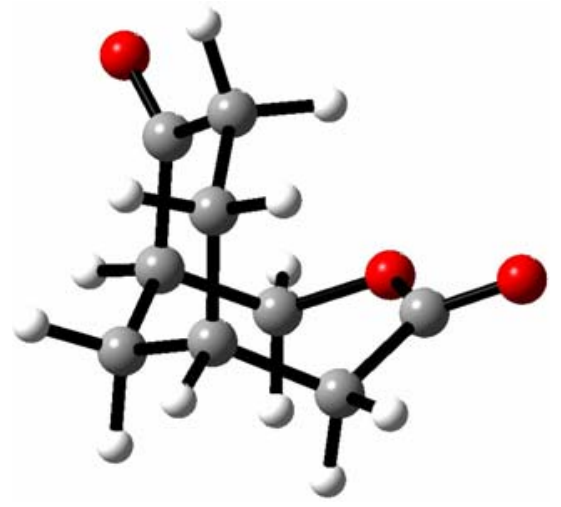

Figure 3: B3PW91/TZ2P Structure of the CC conformation of $(1 \mathrm{R}, 6 \mathrm{~S})-2 \mathrm{c}$.

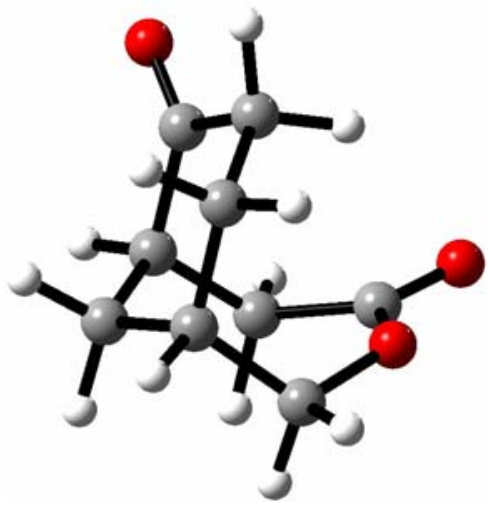

Figure 4: B3PW91/TZ2P Structure of the CC conformation of (1S,6R)-2d. 
Table 1: Dihedral angles of the CC conformations of 2a-2d at the B3LYP/6-31G*, B3LYP/TZ2P and B3PW91/TZ2P levels. ${ }^{\mathrm{a}}$

\begin{tabular}{|c|c|c|c|}
\hline & & $2 a$ & \\
\hline Fnal. & & & 33PW91 \\
\hline Basis Set & $6-31 G^{*}$ & & \\
\hline C1C2O3C4 & 17.2 & 14.5 & 12.8 \\
\hline C2O3C4C5 & -70.3 & -69.1 & -69.2 \\
\hline O3C4C5C6 & 73.5 & 74.0 & 75.1 \\
\hline C4C5C6C7 & 63.6 & 63.5 & 63.5 \\
\hline C5C6C7C8 & -73.8 & -74.0 & -73.4 \\
\hline C6C7C8C9 & -43.2 & -41.3 & -40.9 \\
\hline C7C8C9C1 & 36.7 & 33.7 & 32.6 \\
\hline C8C9C1C2 & 91.9 & 93.9 & 94.8 \\
\hline C9C1C2O3 & -80.1 & -77.9 & -76.1 \\
\hline $\mathrm{C} 10 \mathrm{C} 1 \mathrm{C} 2 \mathrm{O} 3$ & 50.2 & 52.5 & 54.4 \\
\hline C10C6C5C4 & -61.2 & -61.2 & -61.0 \\
\hline C10C1C9C8 & -39.5 & -37.3 & -36.3 \\
\hline C10C6C7C8 & 52.5 & 52.3 & 52.8 \\
\hline C2C1C10C6 & -80.5 & -81.1 & -81.5 \\
\hline C5C6C10C1 & 69.9 & 68.4 & 67.6 \\
\hline C9C1C10C6 & 50.9 & 50.3 & 50.1 \\
\hline C7C6C10C1 & -57.5 & -57.9 & -58.4 \\
\hline
\end{tabular}

\begin{tabular}{|c|c|c|c|}
\hline & & $2 b$ & \\
\hline Fnal. & & & B3PW91 \\
\hline Basis Set & $6-31 G^{*}$ & & \\
\hline $\mathrm{C} 1 \mathrm{O} 2 \mathrm{C} 3 \mathrm{C} 4$ & 9.7 & 11.6 & 10.6 \\
\hline $\mathrm{O} 2 \mathrm{C} 3 \mathrm{C} 4 \mathrm{C} 5$ & -62.0 & -63.4 & -63.9 \\
\hline С3C4C5C6 & 70.5 & 70.7 & 71.5 \\
\hline $\mathrm{C} 4 \mathrm{C} 5 \mathrm{C} 6 \mathrm{C} 7$ & 63.4 & 63.8 & 63.7 \\
\hline C5C6C7C8 & -74.0 & -74.7 & -74.3 \\
\hline C6C7C8C9 & -43.2 & -42.3 & -41.8 \\
\hline C7C8C9C1 & 35.8 & 35.1 & 34.2 \\
\hline $\mathrm{C} 8 \mathrm{C} 9 \mathrm{C} 1 \mathrm{O} 2$ & 92.7 & 93.1 & 94.1 \\
\hline $\mathrm{C} 9 \mathrm{C} 1 \mathrm{O} 2 \mathrm{C} 3$ & -77.3 & -78.7 & -77.4 \\
\hline $\mathrm{C} 10 \mathrm{C} 1 \mathrm{O} 2 \mathrm{C} 3$ & 53.2 & 52.1 & 53.5 \\
\hline C10C6C5C4 & -61.5 & -61.3 & -61.2 \\
\hline $\mathrm{C} 10 \mathrm{C} 1 \mathrm{C} 9 \mathrm{C} 8$ & -38.2 & -37.9 & -37.1 \\
\hline C10C6C7C8 & 52.4 & 51.8 & 52.0 \\
\hline $\mathrm{O} 2 \mathrm{C} 1 \mathrm{C} 10 \mathrm{C} 6$ & -81.3 & -81.5 & -81.9 \\
\hline C5C6C10C1 & 70.2 & 70.4 & 69.8 \\
\hline C9C1C10C6 & 49.4 & 49.3 & 49.1 \\
\hline C7C6C10C1 & -56.3 & -56.1 & -56.4 \\
\hline
\end{tabular}




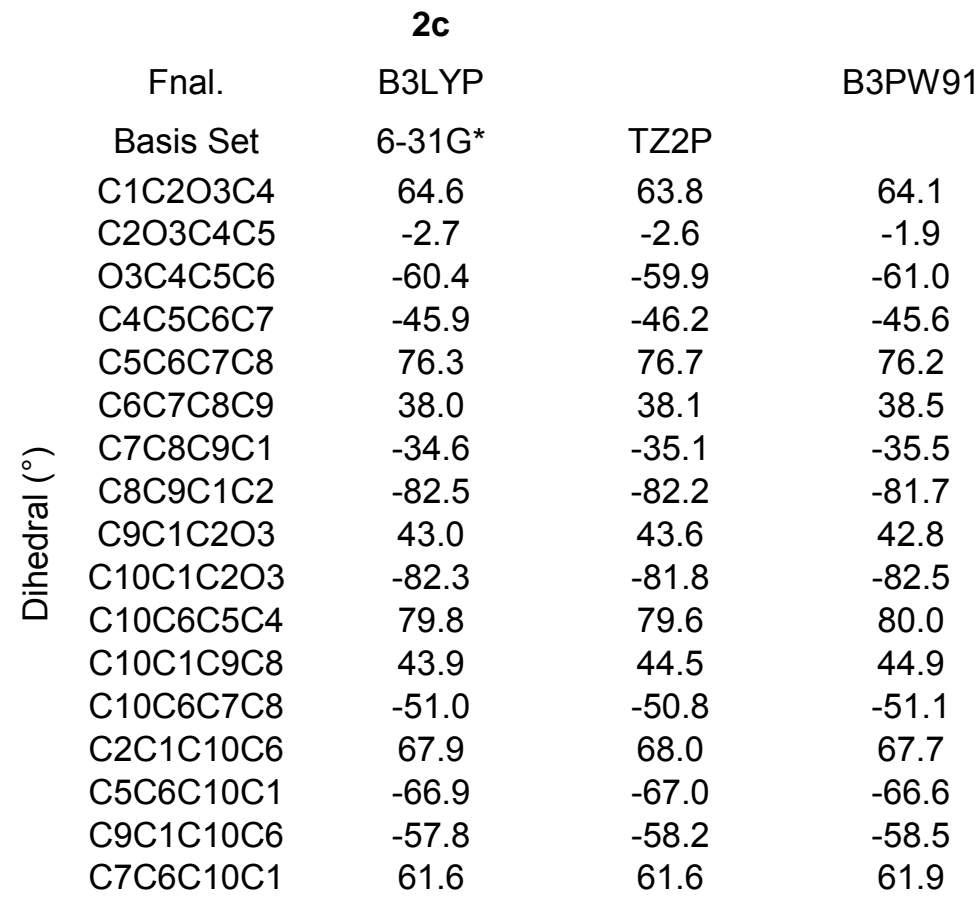

\begin{tabular}{cccc} 
Fnal. & \multicolumn{2}{c}{ B3LYP } & \multicolumn{2}{c}{ B3PW91 } \\
Basis Set & $6-31 G^{*}$ & \multicolumn{2}{c}{ TZ2P } \\
C1C2O3C4 & -66.4 & -65.3 & -65.6 \\
C2O3C4C5 & 5.7 & 4.7 & 4.0 \\
O3C4C5C6 & 57.6 & 57.6 & 58.9 \\
C4C5C6C7 & 45.4 & 46.0 & 45.3 \\
C5C6C7C8 & -79.0 & -78.4 & -77.7 \\
C6C7C8C9 & -37.8 & -38.3 & -38.7 \\
C7C8C9C1 & 38.7 & 38.6 & 38.8 \\
C8C9C1C2 & 77.7 & 78.5 & 78.2 \\
$\overline{\widetilde{\varpi}}$ C9C1C2O3 & -44.2 & -44.4 & -43.4 \\
$\bar{\Phi}$ \\
C10C1C2O3 & 82.1 & 82.2 & 83.1 \\
C10C6C5C4 & -79.2 & -78.6 & -79.0 \\
C10C1C9C8 & -50.0 & -49.5 & -49.6 \\
C10C6C7C8 & 47.1 & 48.0 & 48.5 \\
C2C1C10C6 & -67.6 & -68.1 & -67.8 \\
C5C6C10C1 & 67.3 & 67.1 & 66.5 \\
C9C1C10C6 & 61.0 & 60.9 & 61.1 \\
C7C6C10C1 & -58.9 & -59.4 & -58.8
\end{tabular}

a . ACs of 2a-2d are: (1R,6R)-2a, (1R,6S)-2b, (1R,6S)-2c, and (1S,6R)-2d. 


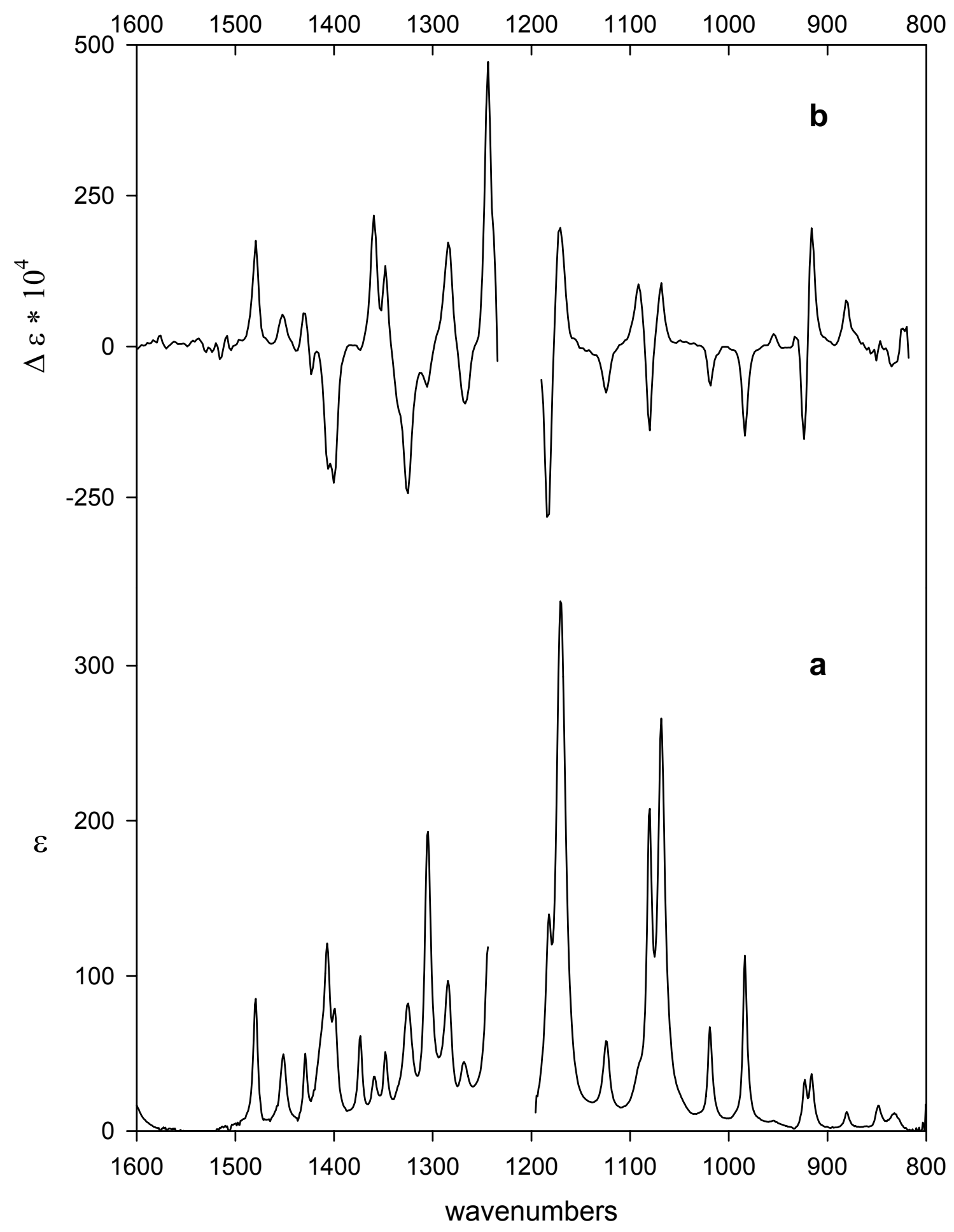

Figure 5: Experimental IR (a) and VCD (b) spectra of a $0.061 \mathrm{M}$ solution of (+)-2 in $\mathrm{CHCl}_{3}$. The gap near $1200 \mathrm{~cm}^{-1}$ is due to $\mathrm{CHCl}_{3}$ absorption. Pathlengths were: a, $239 \mu$; b, $597 \mu$, except in the ranges $912-928,1153-1190,1234-1273$ and $1483-1600 \mathrm{~cm}^{-1}$, where it was $239 \mu$. 
Table 2: Calculated Frequencies, Dipole Strengths and Rotational Strengths for the CC

Conformation of (1R,6R)-2a and Experimental Frequencies, Dipole Strengths and

Rotational Strengths for $(+)-2$.

\begin{tabular}{|c|c|c|c|c|c|c|c|c|c|c|}
\hline \multirow[b]{3}{*}{ mode } & \multicolumn{3}{|c|}{ B3PW91/TZ2P } & \multicolumn{3}{|c|}{ B3LYP/TZ2P } & \multicolumn{4}{|c|}{ Expt. $^{d}$} \\
\hline & \multirow[b]{2}{*}{$v^{a}$} & \multirow[b]{2}{*}{$D^{\mathrm{b}}$} & \multirow[b]{2}{*}{$R^{\mathrm{c}}$} & \multirow[b]{2}{*}{$v^{a}$} & \multirow[b]{2}{*}{$D^{\mathrm{b}}$} & \multirow[b]{2}{*}{$R^{\mathrm{c}}$} & \multicolumn{2}{|c|}{ IR } & \multicolumn{2}{|c|}{ VCD } \\
\hline & & & & & & & $v^{a}$ & $D^{\mathrm{b}}$ & $v^{a}$ & $R^{\mathrm{c}}$ \\
\hline 52 & 1519 & 40.0 & 18.7 & 1529 & 32.4 & 14.8 & 1480 & 43.5 & 1480 & 28.1 \\
\hline 51 & 1493 & 30.2 & 7.3 & 1505 & 26.5 & 6.2 & 1451 & 33.7 & 1452 & 10.1 \\
\hline 50 & 1467 & 23.4 & 7.4 & 1478 & 21.9 & 6.2 & 1429 & 17.2 & 1430 & 12.4 \\
\hline 49 & 1453 & 41.8 & -0.7 & 1466 & 38.6 & -1.2 & 1414 & 30.2 & 1423 & -4.4 \\
\hline 48 & 1445 & 48.4 & -10.1 & 1458 & 42.6 & -6.8 & 1407 & 79.0 & 1406 & -30.1 \\
\hline 47 & 1428 & 26.0 & -25.4 & 1432 & 17.4 & -14.0 & 1399 & 34.2 & 1400 & -35.0 \\
\hline 46 & 1400 & 26.3 & -3.4 & 1408 & 16.2 & -2.3 & 1374 & 30.8 & 1372 & -1.3 \\
\hline 45 & 1387 & 18.6 & 27.0 & 1393 & 12.1 & 3.6 & 1350 & 197 & 1360 & 38.6 \\
\hline 44 & 1384 & 0.8 & 1.8 & 1389 & 5.0 & 6.7 & & & 1357 & 2.6 \\
\hline 43 & 1378 & 32.7 & 22.7 & 1382 & 18.3 & 24.8 & 1348 & 23.8 & 1348 & 21.7 \\
\hline 42 & 1363 & 3.3 & -8.9 & 1371 & 12.3 & -8.0 & 1333 & 12.3 & 1336 & -10.4 \\
\hline 41 & 1351 & 72.0 & -57.5 & 1350 & 17.7 & -16.8 & 1325 & 69.7 & 1326 & -72.7 \\
\hline 40 & 1332 & 171.8 & -4.6 & 1332 & 169.3 & -17.2 & 1305 & 153.7 & 1306 & -12.1 \\
\hline 39 & 1313 & 55.4 & 38.9 & 1312 & 44.9 & 29.6 & 1285 & 91.2 & 1284 & 58.7 \\
\hline 38 & 1289 & 53.5 & -38.0 & 1288 & 54.4 & -47.1 & 1267 & 40.1 & 1268 & -50.6 \\
\hline 37 & 1270 & 47.0 & 76.9 & 1269 & 56.2 & 65.7 & & & 1244 & 96.9 \\
\hline 36 & 1248 & 116.9 & -1.3 & 1248 & 100.2 & 3.3 & & & & \\
\hline 35 & 1234 & 3.2 & -8.4 & 1240 & 5.1 & -9.4 & & & & \\
\hline 34 & 1201 & 38.3 & -24.3 & 1200 & 27.9 & -4.7 & 1183 & 60.9 & 1183 & -82.8 \\
\hline 33 & 1189 & 409.1 & 6.5 & 1178 & 466.0 & -22.6 & 1170 & 452.6 & 1171 & 75.8 \\
\hline 32 & 1140 & 42.2 & -16.1 & 1139 & 58.6 & -18.7 & 1124 & 55.1 & 1125 & -26.2 \\
\hline 31 & 1108 & 73.1 & -15.1 & 1097 & 52.9 & 66.8 & 1095 & 11.5 & 1095 & -2.8 \\
\hline 30 & 1103 & 181.0 & 41.0 & 1096 & 46.5 & -35.2 & 1090 & 14.3 & 1091 & 44.4 \\
\hline 29 & 1093 & 21.3 & -31.9 & 1085 & 148.3 & 7.1 & 1080 & 139.7 & 1081 & -36.0 \\
\hline 28 & 1090 & 122.5 & 41.0 & 1081 & 221.5 & 20.1 & 1068 & 303.0 & 1069 & 29.2 \\
\hline 27 & 1034 & 49.3 & -18.2 & 1033 & 68.2 & -29.1 & 1019 & 53.0 & 1019 & -12.8 \\
\hline 26 & 999 & 92.1 & -22.6 & 994 & 81.3 & -26.6 & 984 & 85.3 & 984 & -33.3 \\
\hline 25 & 968 & 0.4 & -2.1 & 962 & 3.9 & 9.6 & 955 & 14.4 & 955 & 5.3 \\
\hline 24 & 935 & 13.4 & -37.1 & 933 & 7.3 & -23.6 & 923 & 18.5 & 924 & -65.8 \\
\hline 23 & 927 & 40.1 & 52.6 & 924 & 47.7 & 26.8 & 916 & 28.9 & 916 & 69.7 \\
\hline 22 & 891 & 7.6 & 16.9 & 887 & 11.3 & 22.5 & 880 & 9.1 & 881 & 21.3 \\
\hline 21 & 859 & 24.4 & -2.1 & 856 & 23.8 & -5.5 & 848 & 15.9 & & \\
\hline 20 & 842 & 17.3 & -7.9 & 842 & 22.0 & -10.2 & 832 & 19.1 & & \\
\hline . & & & & & & & & & & \\
\hline & & & & & & & & & & \\
\hline & & & & & & & & & & \\
\hline & & & & & & & & & & \\
\hline
\end{tabular}


Table 3: Calculated Frequencies, Dipole Strengths and Rotational Strengths for the CC Conformation of (1R,6S)-2b.

\begin{tabular}{|c|c|c|c|c|c|c|}
\hline \multirow[b]{2}{*}{ mode } & \multicolumn{3}{|c|}{ B3PW91/TZ2P } & \multicolumn{3}{|c|}{ B3LYP/TZ2P } \\
\hline & $v$ & $D$ & $R$ & $v$ & $D$ & $R$ \\
\hline 52 & 1489 & 27.7 & -8.0 & 1500 & 23.6 & -4.6 \\
\hline 51 & 1481 & 47.2 & 16.5 & 1496 & 40.9 & 14.0 \\
\hline 50 & 1471 & 21.7 & -2.4 & 1483 & 23.2 & -2.0 \\
\hline 49 & 1451 & 44.1 & -4.7 & 1465 & 40.1 & -3.6 \\
\hline 48 & 1439 & 23.9 & 35.4 & 1449 & 22.3 & 16.1 \\
\hline 47 & 1428 & 98.8 & -13.1 & 1432 & 73.7 & 3.6 \\
\hline 46 & 1398 & 8.5 & 6.5 & 1406 & 10.0 & 6.2 \\
\hline 45 & 1391 & 11.7 & -24.0 & 1396 & 4.9 & -7.0 \\
\hline 44 & 1388 & 35.8 & -33.8 & 1394 & 12.4 & -20.1 \\
\hline 43 & 1378 & 35.9 & -36.8 & 1386 & 42.7 & -43.8 \\
\hline 42 & 1362 & 64.1 & -29.8 & 1361 & 36.7 & -25.6 \\
\hline 41 & 1351 & 7.9 & 14.6 & 1356 & 17.7 & 6.2 \\
\hline 40 & 1320 & 11.2 & -0.5 & 1320 & 10.3 & 11.4 \\
\hline 39 & 1308 & 66.2 & 10.4 & 1309 & 57.4 & -6.2 \\
\hline 38 & 1276 & 70.5 & -61.9 & 1275 & 29.2 & -38.1 \\
\hline 37 & 1259 & 191.7 & -129.7 & 1256 & 154.6 & -128.5 \\
\hline 36 & 1245 & 85.9 & 21.1 & 1247 & 96.7 & 12.8 \\
\hline 35 & 1235 & 57.1 & 78.8 & 1240 & 46.5 & 61.7 \\
\hline 34 & 1197 & 216.9 & 13.2 & 1194 & 136.6 & 3.8 \\
\hline 33 & 1154 & 446.6 & -43.4 & 1152 & 593.5 & -94.4 \\
\hline 32 & 1132 & 78.4 & 111.2 & 1131 & 121.1 & 146.3 \\
\hline 31 & 1114 & 153.3 & 26.3 & 1111 & 145.6 & 25.8 \\
\hline 30 & 1095 & 16.6 & -2.6 & 1090 & 30.1 & -15.8 \\
\hline 29 & 1087 & 25.6 & -21.8 & 1083 & 6.6 & -15.6 \\
\hline 28 & 1063 & 168.8 & -32.4 & 1049 & 200.7 & -25.9 \\
\hline 27 & 1003 & 22.6 & 21.6 & 1001 & 35.2 & 35.6 \\
\hline 26 & 991 & 59.5 & 6.8 & 985 & 124.0 & -1.8 \\
\hline 25 & 958 & 16.9 & -5.6 & 959 & 19.7 & -6.3 \\
\hline 24 & 947 & 15.6 & 26.7 & 938 & 18.1 & 29.4 \\
\hline 23 & 911 & 24.5 & 20.6 & 912 & 16.8 & 13.5 \\
\hline 22 & 886 & 29.5 & 17.6 & 887 & 37.6 & 14.5 \\
\hline 21 & 854 & 34.5 & -56.9 & 850 & 28.6 & -50.7 \\
\hline 20 & 824 & 1.1 & 5.3 & 820 & 3.3 & 8.2 \\
\hline
\end{tabular}


Table 4: Calculated Frequencies, Dipole Strengths and Rotational Strengths for the CC conformation of (1R,6S)-2c.

\begin{tabular}{|c|c|c|c|c|c|c|}
\hline \multirow[b]{2}{*}{ mode } & \multicolumn{3}{|c|}{ B3PW91/TZ2P } & \multicolumn{3}{|c|}{ B3LYP/TZ2P } \\
\hline & $v$ & $D$ & $R$ & $v$ & $D$ & $R$ \\
\hline 52 & 1507 & 16.8 & -6.5 & 1518 & 13.6 & -2.4 \\
\hline 51 & 1498 & 59.2 & -10.8 & 1508 & 50.0 & -7.0 \\
\hline 50 & 1486 & 18.9 & 7.1 & 1497 & 15.4 & 4.0 \\
\hline 49 & 1470 & 58.2 & 12.5 & 1482 & 47.5 & 8.8 \\
\hline 48 & 1458 & 35.1 & 1.6 & 1471 & 31.6 & 3.6 \\
\hline 47 & 1418 & 35.1 & 19.7 & 1422 & 21.9 & 17.2 \\
\hline 46 & 1394 & 42.6 & -24.6 & 1401 & 44.8 & -16.8 \\
\hline 45 & 1391 & 12.7 & 8.8 & 1398 & 6.0 & 6.9 \\
\hline 44 & 1373 & 13.9 & -14.9 & 1382 & 5.9 & -7.6 \\
\hline 43 & 1367 & 2.6 & -3.1 & 1368 & 2.4 & -4.6 \\
\hline 42 & 1354 & 79.2 & -77.2 & 1356 & 35.9 & -37.2 \\
\hline 41 & 1343 & 52.6 & -5.8 & 1345 & 69.9 & -32.3 \\
\hline 40 & 1320 & 27.7 & -6.4 & 1323 & 33.9 & -6.1 \\
\hline 39 & 1312 & 42.2 & -12.8 & 1310 & 37.0 & -5.4 \\
\hline 38 & 1274 & 127.1 & -74.1 & 1274 & 83.6 & -66.7 \\
\hline 37 & 1265 & 57.5 & 32.2 & 1267 & 42.2 & 8.7 \\
\hline 36 & 1255 & 39.5 & 20.4 & 1257 & 36.5 & 8.6 \\
\hline 35 & 1242 & 63.0 & 86.0 & 1241 & 61.9 & 91.9 \\
\hline 34 & 1190 & 365.2 & -130.1 & 1190 & 104.0 & -100.6 \\
\hline 33 & 1176 & 171.4 & 103.0 & 1175 & 485.1 & 35.8 \\
\hline 32 & 1142 & 79.9 & 17.7 & 1136 & 124.0 & 18.5 \\
\hline 31 & 1122 & 43.7 & -19.5 & 1113 & 27.8 & -16.3 \\
\hline 30 & 1099 & 116.1 & 9.7 & 1094 & 73.3 & -24.7 \\
\hline 29 & 1087 & 50.0 & 38.8 & 1079 & 71.9 & 62.6 \\
\hline 28 & 1068 & 105.7 & 30.7 & 1059 & 185.6 & 54.5 \\
\hline 27 & 1020 & 45.0 & 1.9 & 1015 & 50.4 & -12.4 \\
\hline 26 & 998 & 29.5 & 64.2 & 997 & 54.4 & 85.7 \\
\hline 25 & 976 & 46.5 & 29.6 & 971 & 45.2 & 35.6 \\
\hline 24 & 965 & 17.3 & -4.7 & 961 & 18.0 & -11.7 \\
\hline 23 & 920 & 15.9 & -8.2 & 913 & 20.4 & -9.6 \\
\hline 22 & 902 & 8.7 & 10.7 & 907 & 8.0 & 8.3 \\
\hline 21 & 865 & 9.4 & -1.1 & 854 & 18.4 & -11.9 \\
\hline 20 & 852 & 72.5 & -43.9 & 850 & 67.7 & -31.2 \\
\hline
\end{tabular}


Table 5: Calculated Frequencies, Dipole Strengths and Rotational Strengths for the CC conformation of (1S,6R)-2d.

\begin{tabular}{|c|c|c|c|c|c|c|}
\hline \multirow[b]{2}{*}{ mode } & \multicolumn{3}{|c|}{ B3PW91/TZ2P } & \multicolumn{3}{|c|}{ B3LYP/TZ2P } \\
\hline & $v$ & $D$ & $R$ & $v$ & $D$ & $R$ \\
\hline 52 & 1505 & 29.8 & 13.6 & 1515 & 20.6 & 8.5 \\
\hline 51 & 1494 & 34.6 & 5.3 & 1504 & 34.7 & 2.9 \\
\hline 50 & 1484 & 42.4 & 6.2 & 1496 & 36.5 & 7.5 \\
\hline 49 & 1469 & 33.3 & 6.3 & 1482 & 28.9 & 6.2 \\
\hline 48 & 1464 & 44.1 & -12.0 & 1477 & 35.1 & -9.3 \\
\hline 47 & 1422 & 37.6 & -9.2 & 1427 & 24.7 & -7.0 \\
\hline 46 & 1391 & 7.7 & -2.1 & 1397 & 13.2 & 0.9 \\
\hline 45 & 1385 & 25.6 & 29.1 & 1389 & 13.5 & 25.6 \\
\hline 44 & 1376 & 3.4 & -6.9 & 1381 & 4.9 & -5.0 \\
\hline 43 & 1370 & 9.1 & -14.9 & 1378 & 11.4 & -8.7 \\
\hline 42 & 1365 & 61.3 & 86.1 & 1365 & 37.6 & 57.2 \\
\hline 41 & 1333 & 32.1 & -49.6 & 1335 & 21.9 & -36.4 \\
\hline 40 & 1327 & 64.1 & 3.6 & 1330 & 57.5 & 11.7 \\
\hline 39 & 1304 & 45.8 & 3.1 & 1304 & 43.8 & 0.1 \\
\hline 38 & 1279 & 7.4 & -2.1 & 1281 & 3.7 & 6.2 \\
\hline 37 & 1263 & 170.7 & -37.2 & 1264 & 92.1 & -19.4 \\
\hline 36 & 1253 & 145.2 & 67.1 & 1251 & 97.4 & 58.4 \\
\hline 35 & 1244 & 29.9 & -20.1 & 1243 & 96.5 & -32.5 \\
\hline 34 & 1194 & 174.5 & -104.6 & 1195 & 88.1 & -83.9 \\
\hline 33 & 1176 & 207.7 & 76.2 & 1175 & 363.1 & 61.2 \\
\hline 32 & 1139 & 30.1 & -6.4 & 1130 & 35.0 & -3.4 \\
\hline 31 & 1117 & 57.5 & -13.3 & 1114 & 98.7 & 26.9 \\
\hline 30 & 1107 & 167.2 & 131.7 & 1097 & 121.5 & 83.9 \\
\hline 29 & 1078 & 16.4 & -9.7 & 1071 & 22.2 & 0.7 \\
\hline 28 & 1067 & 162.3 & -91.8 & 1056 & 263.8 & -97.8 \\
\hline 27 & 1019 & 64.4 & -10.8 & 1017 & 47.0 & 6.3 \\
\hline 26 & 1010 & 5.1 & -12.6 & 1008 & 23.0 & -39.8 \\
\hline 25 & 966 & 22.8 & -12.8 & 963 & 44.2 & -22.5 \\
\hline 24 & 960 & 42.1 & 20.2 & 956 & 31.4 & 25.1 \\
\hline 23 & 927 & 11.1 & -19.3 & 919 & 9.7 & -17.3 \\
\hline 22 & 905 & 2.6 & -10.0 & 909 & 0.7 & -4.4 \\
\hline 21 & 879 & 37.8 & -9.0 & 869 & 42.3 & -10.4 \\
\hline 20 & 822 & 20.4 & 0.5 & 821 & 22.1 & -0.6 \\
\hline
\end{tabular}



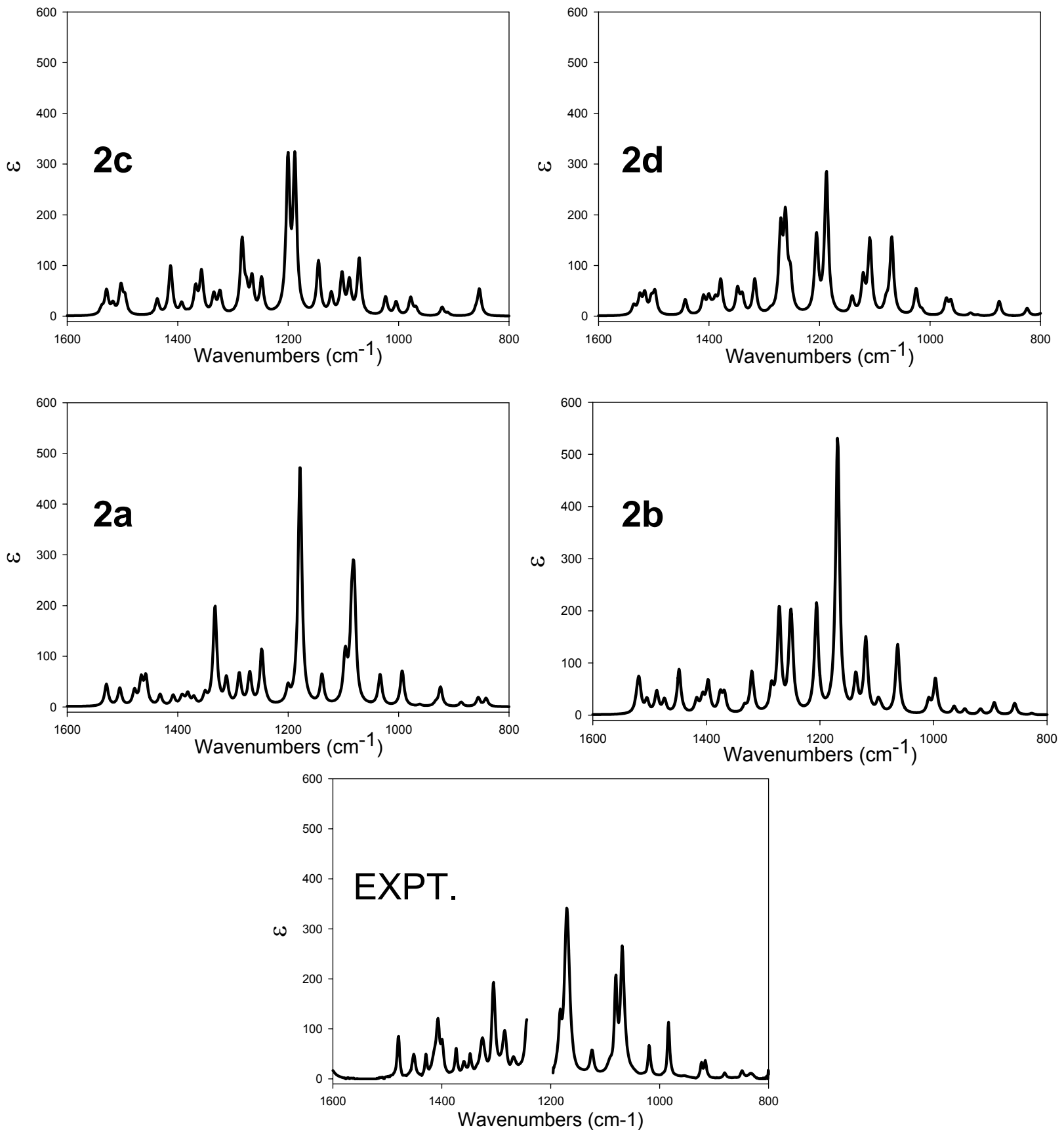

Figure 6: Comparison of the B3LYP/TZ2P IR spectra of 2a-2d to the experimental IR spectrum of $(+)-2$. 

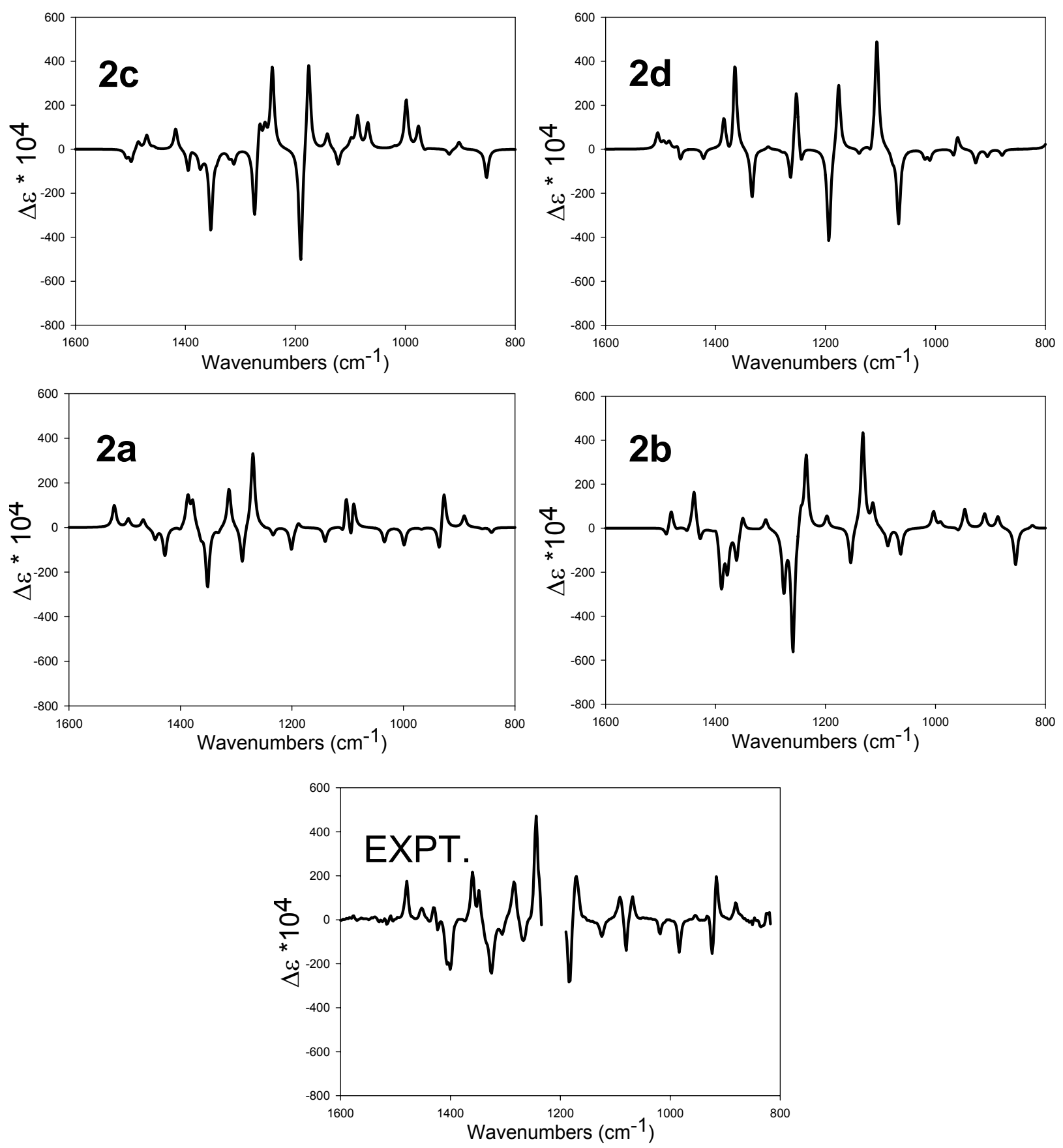

Figure 7: Comparison of the B3LYP/TZ2P VCD spectra of 2a-2d to the experimental VCD spectrum of (+)-2. 


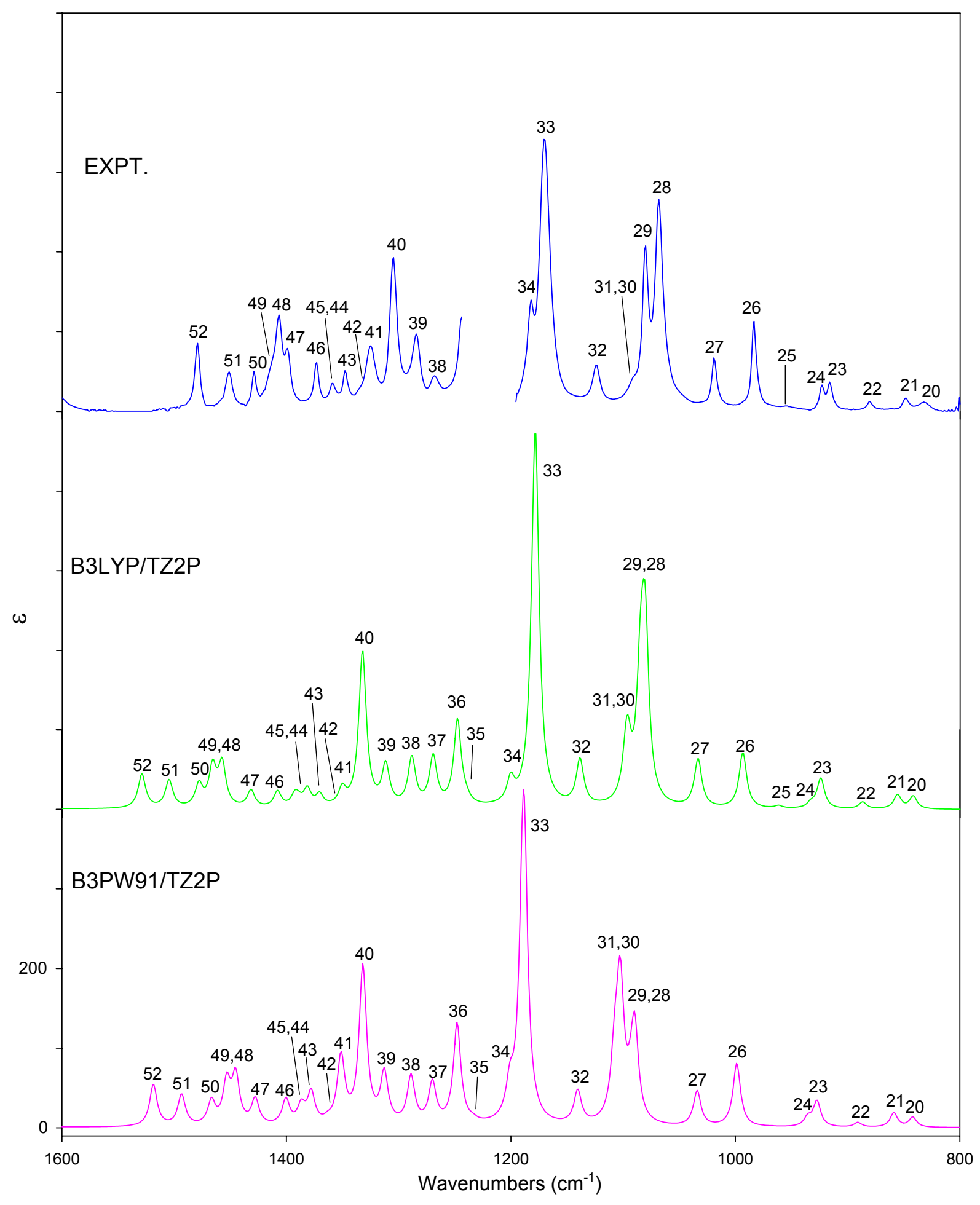

Figure 8: Comparison of the B3PW91/TZ2P and B3LYP/TZ2P IR spectra of 2a to the experimental IR spectrum of $(+)-2$. 


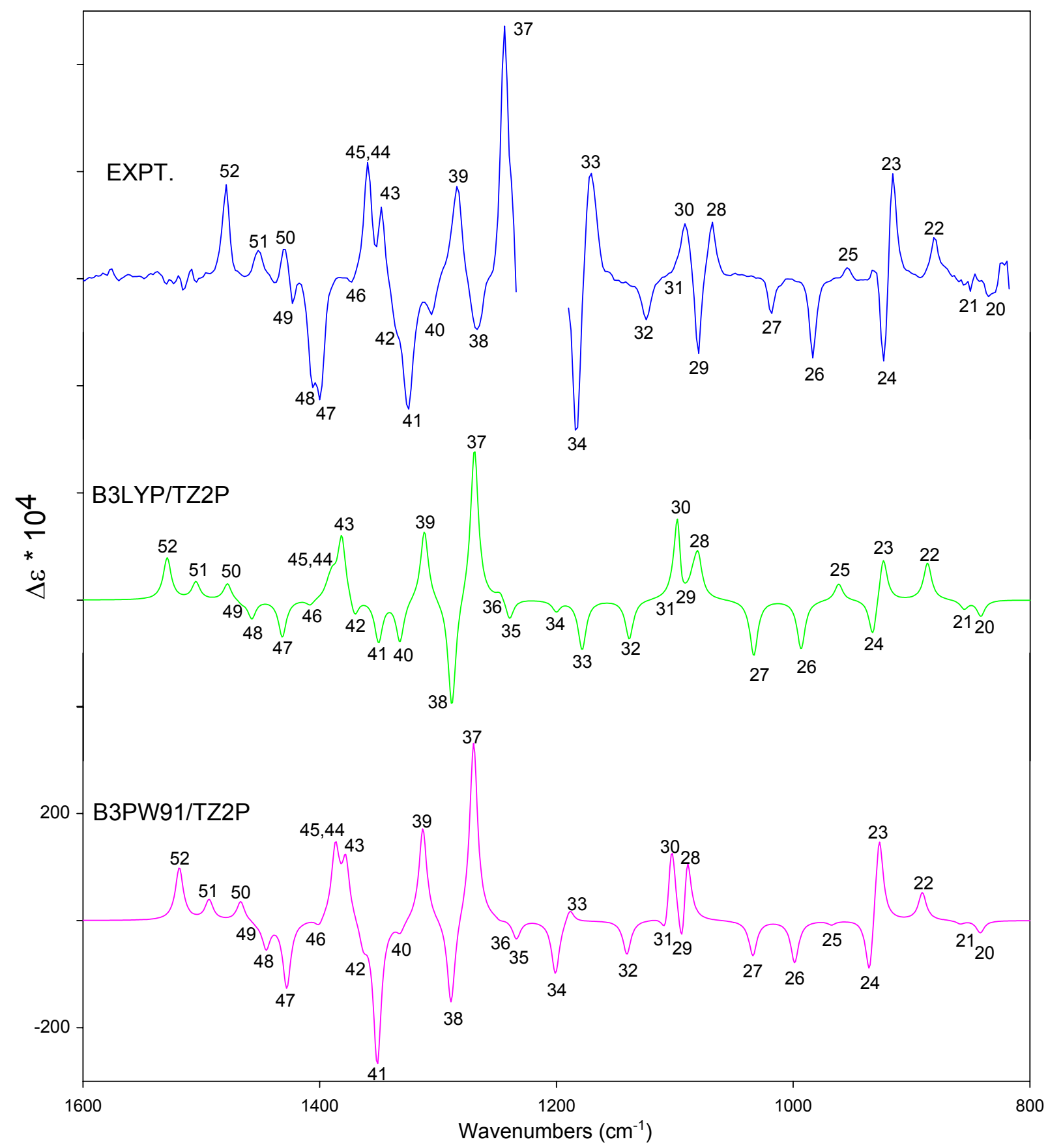

Figure 9: Comparison of the B3PW91/TZ2P and B3LYP/TZ2P VCD spectra of the CC conformation of (1R,6R)-2a to the experimental VCD spectrum of (+)-2. 

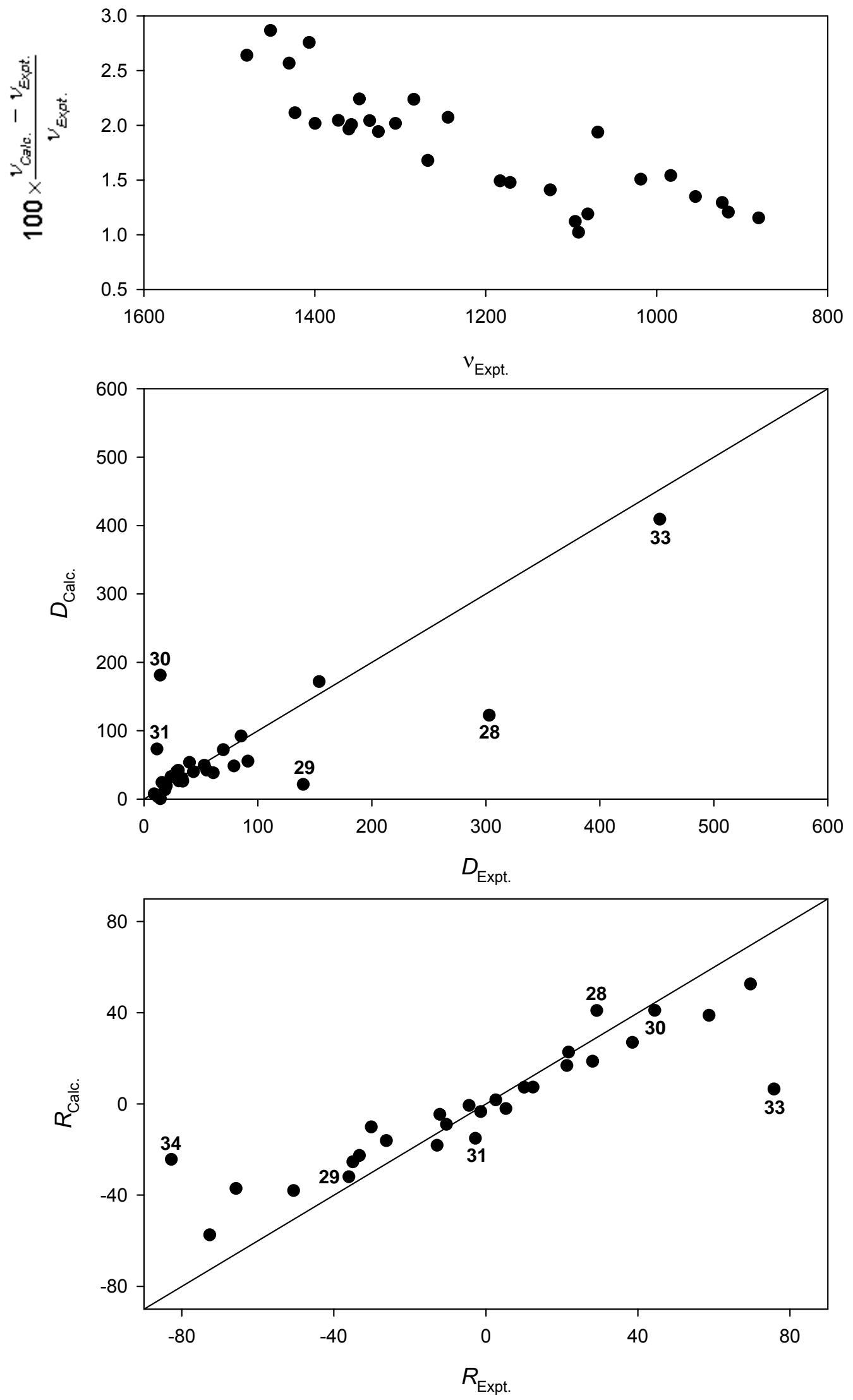
Figure 10: Comparison of B3PW91/TZ2P frequencies (in $\mathrm{cm}^{-1}$ ), dipole strengths (in $10^{-40}$ $\mathrm{esu}^{2} \mathrm{~cm}^{2}$ ) and rotational strengths (in $10^{-44} \mathrm{esu}^{2} \mathrm{~cm}^{2}$ ) for (1R,6R)-2a to the experimental parameters for $(+)-2$. 

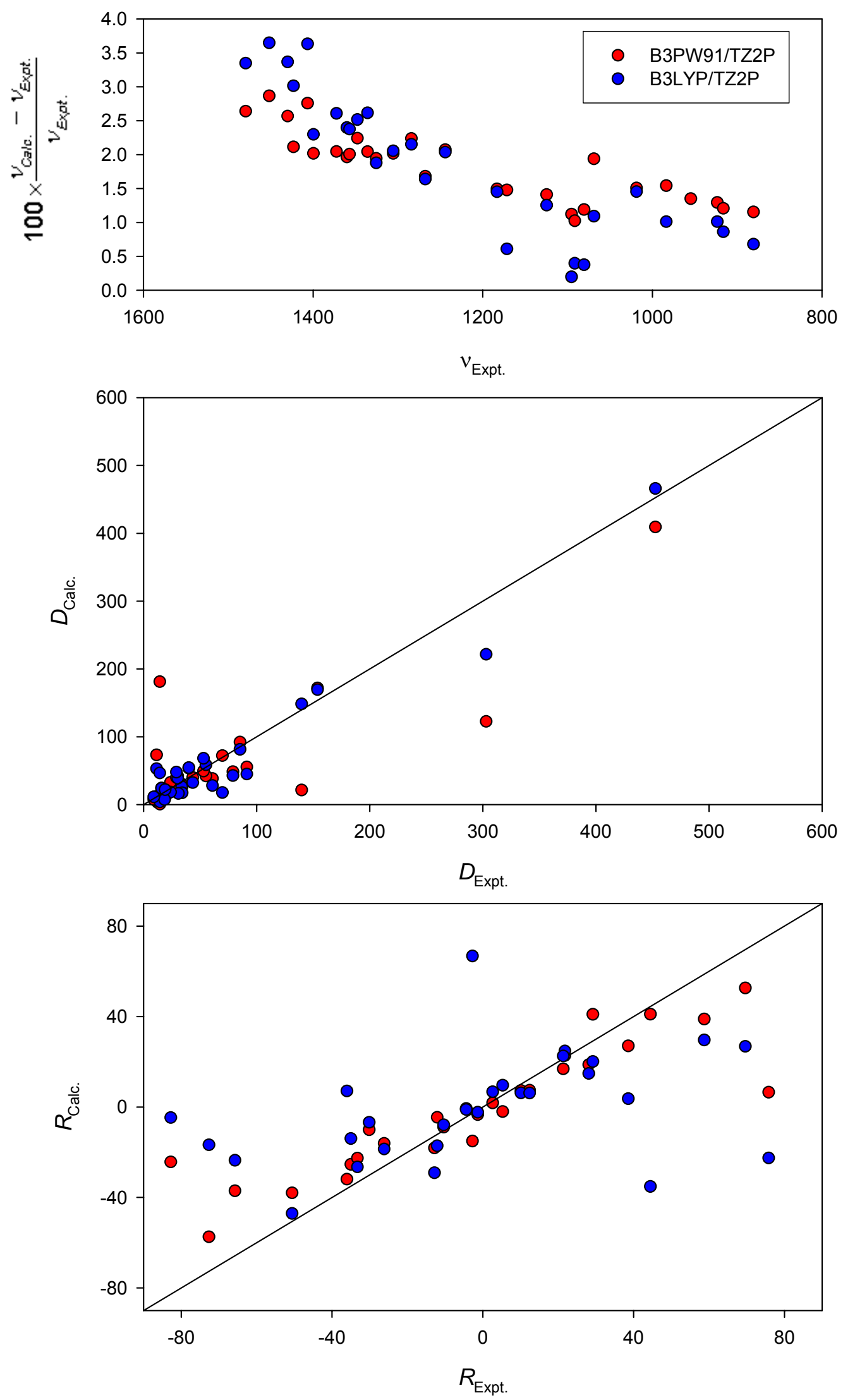
Figure 11: Comparison of B3PW91/TZ2P (•) and B3LYP/TZ2P (•) frequencies (in $\mathrm{cm}^{-}$ ${ }^{1}$ ), dipole strengths (in $10^{-40} \mathrm{esu}^{2} \mathrm{~cm}^{2}$ ) and rotational strengths (in $10^{-44} \mathrm{esu}^{2} \mathrm{~cm}^{2}$ ) for the CC conformation of (1R,6R)-2a to the experimental parameters for $(+)-2$.

Table 6: Observed chemical shifts and coupling constants of 2.

\begin{tabular}{|c|c|c|c|c|c|c|c|}
\hline $\mathrm{H}$ & shift & 1 & 2 & 5 & 6 & 7 & 11 \\
\hline 1 & 1.92 & & & & & & \\
\hline 2 & 2.10 & & & & & & \\
\hline 3 & 2.11 & & (NV) & & & & \\
\hline 4 & 2.15 & 15.5 & & & & & \\
\hline 5 & 2.48 & & & & & & \\
\hline 6 & 2.58 & & & 16.5 & & & \\
\hline 7 & 2.64 & & & & & & \\
\hline 8 & 2.70 & & & & 6.2 & & \\
\hline 9 & 2.83 & & & & & 18.1 & \\
\hline 10 & 3.61 & & & & & 10 & \\
\hline 11 & 4.13 & & & & & & \\
\hline 12 & 4.14 & & & & & & (NV) \\
\hline
\end{tabular}




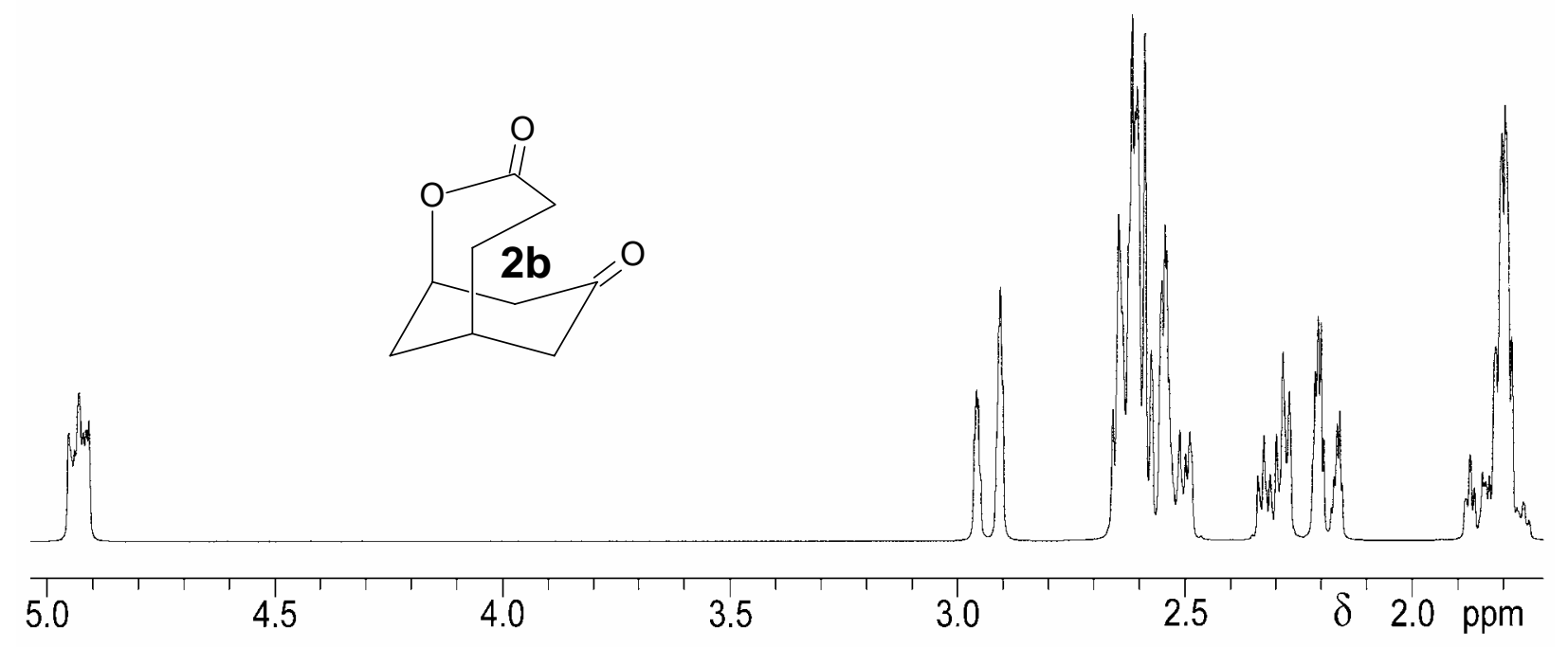

Figure 12: Calculated ${ }^{1} \mathrm{H}$ NMR spectrum of the CC conformation of $\mathbf{2 b}$.

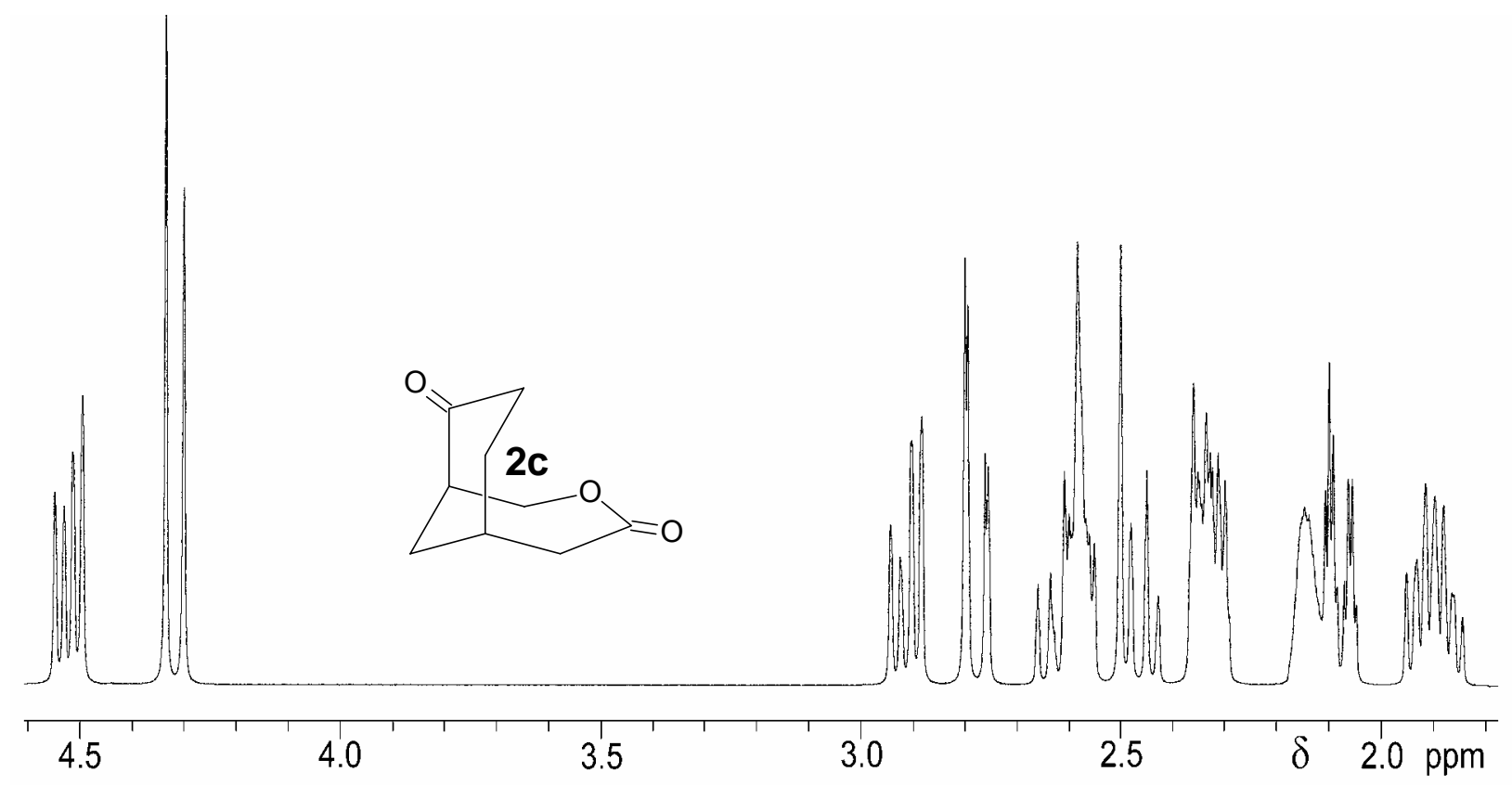

Figure 13: Calculated ${ }^{1} \mathrm{H}$ NMR spectrum of the $\mathrm{CC}$ conformation of $\mathbf{2 c}$. 


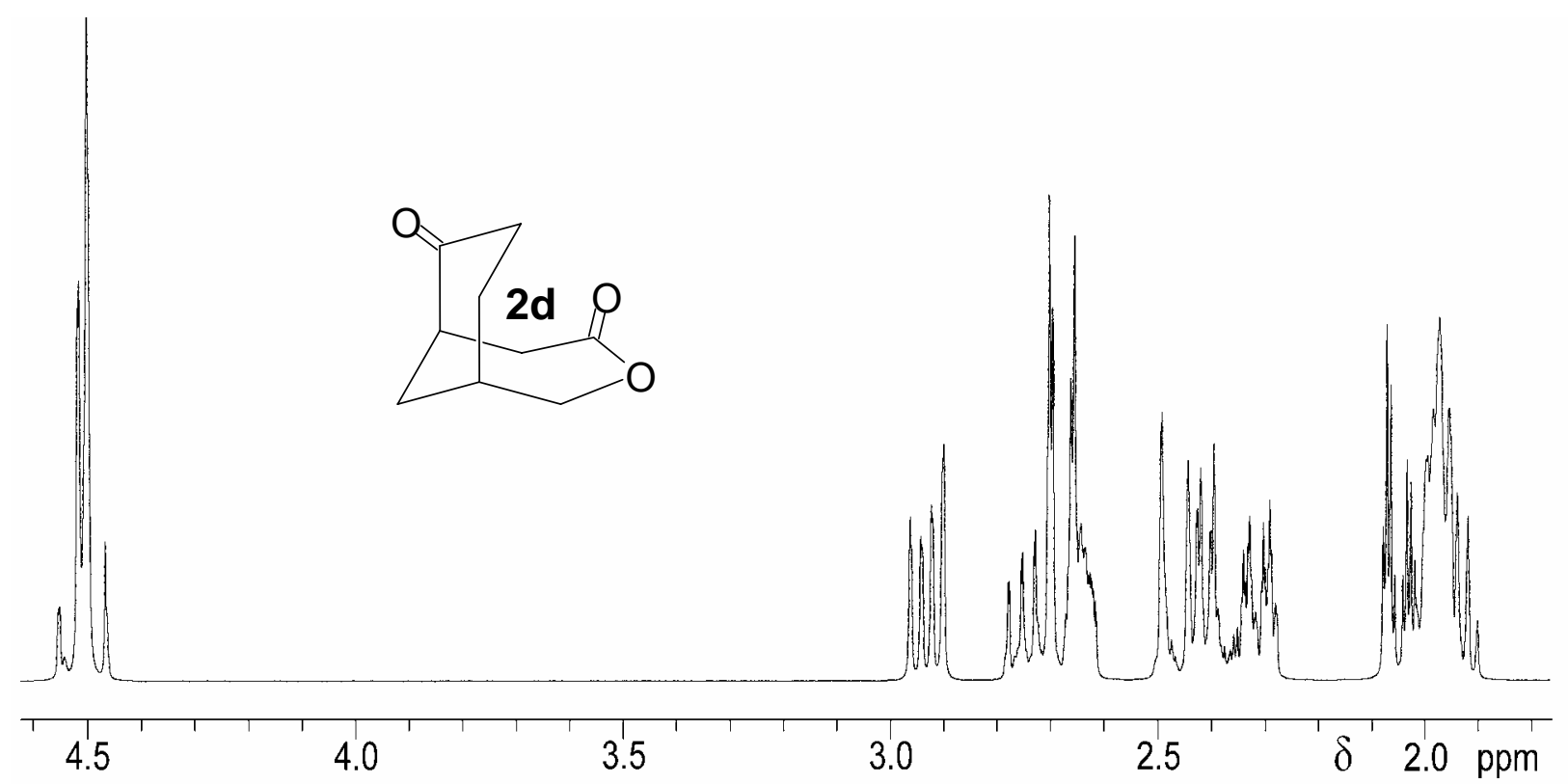

Figure 14: Calculated ${ }^{1} \mathrm{H}$ NMR spectrum of the $\mathrm{CC}$ conformation of $\mathbf{2 d}$.

Table 7: Calculated ${ }^{1} \mathrm{H}$ chemical shifts and coupling constants for the $\mathrm{CC}$ conformation of $2 \mathbf{a}$.

\begin{tabular}{|c|c|c|c|c|c|c|c|c|c|c|c|c|}
\hline & & & & & & & $\mathrm{J}^{\mathrm{a}}$ & & & & & \\
\hline $\begin{array}{l}\mathbf{H} \\
1\end{array}$ & Shift & 1 & 2 & 3 & 4 & 5 & 6 & 7 & 8 & 9 & 10 & 11 \\
\hline $\begin{array}{l}1 \\
2\end{array}$ & $\begin{array}{l}.00 \\
2.08\end{array}$ & 1.63 & & & & & & & & & & \\
\hline 3 & 2.12 & -0.33 & -15.86 & & & & & & & & & \\
\hline 4 & 2.16 & -16.54 & -0.46 & 0.35 & & & & & & & & \\
\hline 5 & 2.48 & 0.36 & -0.30 & 2.18 & -0.30 & & & & & & & \\
\hline 6 & 2.59 & -0.43 & 0.38 & -0.16 & 0.79 & -17.30 & & & & & & \\
\hline 7 & 2.55 & -0.27 & 0.21 & -0.82 & -0.28 & -0.78 & 1.09 & & & & & \\
\hline 8 & 2.7 & 4.25 & 5.07 & 3.05 & 4.18 & 2.17 & 7.31 & -0.32 & & & & \\
\hline 9 & 2.84 & -0.29 & -0.73 & 1.64 & -0.33 & 1.89 & -0.61 & -19.28 & 0.06 & & & \\
\hline 10 & 3.6 & -0.56 & 6.70 & 2.15 & -0.23 & 0.01 & -0.16 & 11.58 & 0.97 & 0.92 & & \\
\hline 11 & 4.02 & 4.80 & -0.41 & -0.24 & 3.52 & -0.12 & -0.78 & 0.31 & 0.59 & -0.16 & -0.70 & \\
\hline 12 & 4.29 & 1.03 & -0.33 & -0.27 & 12.89 & 0.34 & -0.37 & 0.09 & -0.98 & 0.43 & -0.38 & -15.69 \\
\hline
\end{tabular}




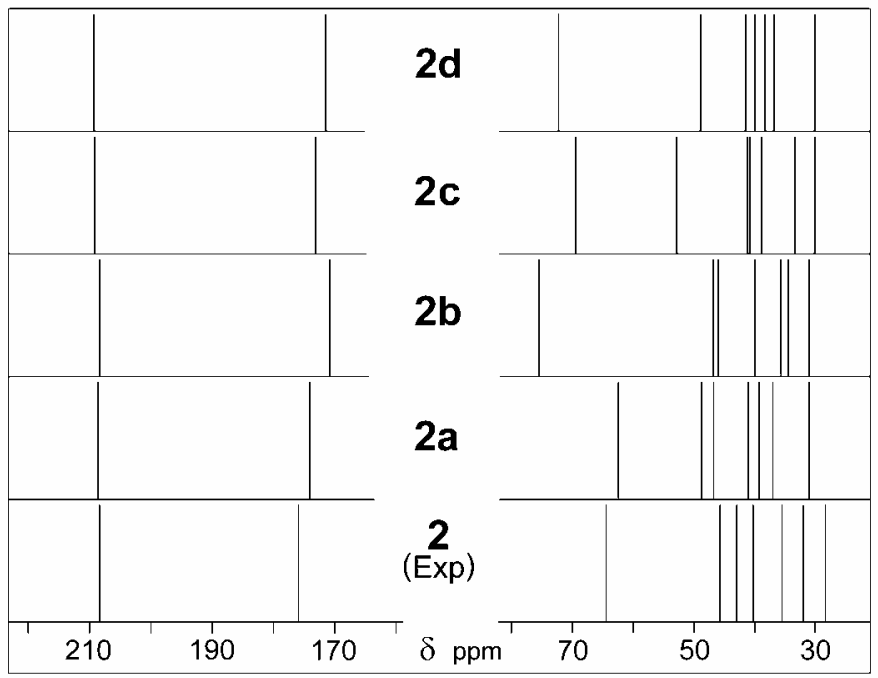

Figure 15: Calculated ${ }^{13} \mathrm{C}$ chemical shifts of the $\mathrm{CC}$ conformations of 2a-2d compared with the experimental ${ }^{13} \mathrm{C}$ chemical shifts of 2 . 
Table 8: Calculated Specific Rotations, Excitation Energies and Rotational Strengths for 2b-2d.

\begin{tabular}{|c|c|c|c|c|c|c|}
\hline & {$[\alpha]_{D}$} & {$[\alpha]_{546}$} & $\Delta \mathrm{E}(\mathrm{eV})$ & $\lambda(\mathrm{nm})$ & $\mathrm{R}_{\mathrm{vel}}{ }^{\mathrm{d}}$ & $R_{\text {length }}{ }^{d}$ \\
\hline \multirow[t]{6}{*}{$2 b^{a}$} & 29 & 35 & 4.15 & 298 & -0.9 & -0.9 \\
\hline & & & 5.41 & 229 & 5.4 & 5.2 \\
\hline & & & 5.54 & 224 & 1.6 & 1.7 \\
\hline & & & 5.74 & 216 & 5.7 & 5.5 \\
\hline & & & 6.15 & 201 & 9.9 & 10.2 \\
\hline & & & 6.19 & 200 & 5.1 & 4.9 \\
\hline \multirow[t]{6}{*}{$2 c^{b}$} & 234 & 283 & 4.15 & 299 & 5.1 & 5.5 \\
\hline & & & 5.39 & 230 & 2.9 & 3.5 \\
\hline & & & 5.63 & 220 & 3.2 & 3.3 \\
\hline & & & 5.86 & 211 & -2.9 & -3.0 \\
\hline & & & 5.88 & 211 & 1.6 & 1.5 \\
\hline & & & 6.27 & 198 & -11.1 & -11.0 \\
\hline \multirow[t]{6}{*}{$2 d^{c}$} & 54 & 69 & 4.14 & 299 & 4.4 & 4.8 \\
\hline & & & 5.38 & 230 & 2.9 & 2.5 \\
\hline & & & 5.60 & 221 & -6.4 & -6.6 \\
\hline & & & 5.75 & 216 & -5.2 & -5.5 \\
\hline & & & 5.84 & 212 & 1.7 & 1.9 \\
\hline & & & 6.24 & 199 & -12.9 & -13.0 \\
\hline \multicolumn{7}{|c|}{$(1 \mathrm{R}, 6 \mathrm{~S}), \mathrm{CC}$ conformation. } \\
\hline \multicolumn{7}{|c|}{ (1R,6S), CC conformation. } \\
\hline \multicolumn{7}{|c|}{ (1S,6R), CC conformation. } \\
\hline
\end{tabular}


Table 9: Variation of Calculated Specific Rotations and Electronic Excitation Energies and Rotational Strengths of the CC conformation of (1R,6R)-2a with Functional, Basis Set and Geometry.

\begin{tabular}{|c|c|c|c|c|c|c|c|c|c|}
\hline Geometry & Fnal. & Basis Set & $\mathrm{C} 1 \mathrm{C} 2 \mathrm{O} 3 \mathrm{C} 4\left(^{\circ}\right)$ & {$[\alpha]_{D}$} & {$[\alpha]_{546}$} & $\lambda(\mathrm{nm})$ & $\Delta \mathrm{E}(\mathrm{eV})$ & $\mathrm{R}_{1}^{\mathrm{vel}, \mathrm{a}}$ & $\mathrm{R}_{2}^{\mathrm{vel}, \mathrm{a}}$ \\
\hline \multirow[t]{2}{*}{ B3LYP/6-31G* } & B3LYP & aug-cc-pVDZ & 17.2 & -43.8 & -52.6 & 299 & 4.15 & -1.2 & \\
\hline & & & & & & 233 & 5.32 & & 12.5 \\
\hline \multirow[t]{2}{*}{ B3LYP/6-31G* } & B3PW91 & aug-cc-pVDZ & 17.2 & -43.4 & -52.0 & 299 & 4.15 & -1.3 & \\
\hline & & & & & & 232 & 5.34 & & 12.2 \\
\hline \multirow[t]{2}{*}{ B3LYP/6-31G* } & B3LYP & $6-311++G(2 d, 2 p)$ & 17.2 & -44.6 & -53.4 & 298 & 4.16 & -1.1 & \\
\hline & & & & & & 232 & 5.33 & & 12.6 \\
\hline \multirow{2}{*}{ MMFF94 } & R3I YP & 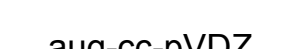 & 611 & $12 \bar{a} 7$ & 1531 & 309 & 101 & 03 & \\
\hline & Dontr & ang-co-pvis & 01.4 & & & 250 & 4.96 & & 11.6 \\
\hline \multirow[t]{2}{*}{ AM1 } & B3LYP & aug-cc-pVDZ & 20.2 & -77.9 & -92.0 & 313 & 3.96 & -0.6 & \\
\hline & & & & & & 250 & 4.96 & & 12.9 \\
\hline \multirow[t]{2}{*}{$\mathrm{HF} / 6-31 \mathrm{G}^{*}$} & B3LYP & aug-cc-pVDZ & 29.9 & -82.7 & -98.7 & 290 & 4.27 & -0.9 & \\
\hline & & & & & & 226 & 5.48 & & 16.5 \\
\hline \multirow{2}{*}{ B3LYP/6-31G* Spartan } & B3LYP & aug-cc-pVDZ & 19.4 & -56.0 & -67.1 & 299 & 4.14 & -1.1 & \\
\hline & & & & & & 234 & 5.31 & & 12.2 \\
\hline \multirow[t]{2}{*}{ B3LYP/TZ2P } & B3LYP & aug-cc-pVDZ & 14.5 & -27.7 & -33.3 & 296 & 4.19 & -1.3 & \\
\hline & & & & & & 230 & 5.39 & & 12.8 \\
\hline \multirow[t]{2}{*}{ BLYP/6-31G* } & B3LYP & aug-cc-pVDZ & 15.7 & -37.0 & -44.5 & 304 & 4.08 & -1.3 & \\
\hline & & & & & & 238 & 5.21 & & 11.7 \\
\hline \multirow[t]{2}{*}{ B3PW91/6-31G* } & B3LYP & aug-cc-pVDZ & 14.8 & -31.8 & -38.2 & 298 & 4.16 & -1.2 & \\
\hline & & & & & & 232 & 5.34 & & 12.7 \\
\hline \multirow[t]{2}{*}{ B3PW91/TZ2P } & B3LYP & aug-cc-pVDZ & 12.2 & -18.0 & -21.7 & 295 & 4.20 & -1.3 & \\
\hline & & & & & & 229 & 5.41 & & 12.8 \\
\hline \multirow[t]{2}{*}{ MP2/6-31G* } & B3LYP & aug-cc-pVDZ & 22.2 & -62.0 & -74.0 & 304 & 4.08 & -1.0 & \\
\hline & & & & & & 238 & 5.22 & & 12.1 \\
\hline MP2/6-311G** & B3LYP & aug-cc-pVDZ & 21.9 & -57.9 & -69.0 & 299 & 4.14 & -1.1 & \\
\hline
\end{tabular}




\begin{tabular}{|c|c|c|c|c|c|c|c|c|}
\hline & & & & & & 234 & 5.30 & \\
\hline B3LYP/6-31G* (Constrained) $)^{b}$ & B3LYP & aug-cc-pVDZ & 0.0 & 21.6 & 25.1 & $\begin{array}{l}299 \\
232\end{array}$ & $\begin{array}{l}4.15 \\
5.34\end{array}$ & -1.7 \\
\hline B3PW91/TZ2P (Constrained) & B3LYP & aug-cc-pVDZ & 0.0 & 26.1 & 30.6 & $\begin{array}{l}295 \\
229\end{array}$ & $\begin{array}{l}4.20 \\
5.42\end{array}$ & -1.6 \\
\hline
\end{tabular}

a. In $10^{-40} \mathrm{esu}^{2} \mathrm{~cm}^{2}$.

${ }^{\mathrm{b}}$. Geometries optimized with the constraint that $\mathrm{C} 1 \mathrm{C} 2 \mathrm{O} 3 \mathrm{C} 4=0$, i.e. the lactone group is planar. 
Geometries of the CC conformations of 2a-2d.

\# b3lyp/6-31G *

$(1 R, 6 R)-2 a$ B3LYP/6-31G* Geometry

$\odot 1$

\begin{tabular}{|c|c|c|c|}
\hline C & $\odot .90711400$ & 1.19526500 & $\odot .94508000$ \\
\hline C & 2.06697400 & ๑. 30695600 & $\odot .43776700$ \\
\hline C & 1. 61240500 & -0.90803000 & -0.36044400 \\
\hline C & $\odot .42036600$ & -1.67655700 & $\odot .2015680 \odot$ \\
\hline C & -0.69645900 & -0.82733000 & 0.87356700 \\
\hline $\mathrm{H}$ & -1.22957700 & -1.49572800 & 1.55423000 \\
\hline $\mathrm{H}$ & 2.78536000 & 0.86765300 & -0.16899000 \\
\hline $\mathrm{H}$ & 1.33472400 & 1.91367800 & 1.65685000 \\
\hline $\mathrm{H}$ & ๑. 38167100 & -0.10298900 & 2.58515100 \\
\hline C & -0.11233300 & 0.33307600 & 1.70614300 \\
\hline $\mathrm{H}$ & -0.92785900 & ๑. 95866600 & 2.08968600 \\
\hline $\mathrm{H}$ & $\odot .02514000$ & -2.33365800 & -0.57875600 \\
\hline $\mathrm{H}$ & 0.83702100 & -2.33462600 & $\odot .97971800$ \\
\hline $\mathrm{H}$ & 2.61451200 & $-\odot .0790540 \odot$ & 1.31168800 \\
\hline 0 & 2.19818600 & -1.28395500 & -1.35837600 \\
\hline C & ๑. $260 \odot 280 \odot$ & 2.02510900 & -0.17928700 \\
\hline $\mathrm{H}$ & 1.03501500 & 2.64247000 & -0.65442600 \\
\hline $\mathrm{H}$ & -0.47137600 & 2.71999400 & $\odot .25356200$ \\
\hline C & -0.43091000 & 1.24260800 & -1.29362400 \\
\hline $\mathrm{H}$ & -0.70922700 & 1.93111300 & -2.09529500 \\
\hline $\mathrm{H}$ & $\odot .22194800$ & ๑ . 48519800 & -1.73839000 \\
\hline C & -1.82758500 & $-0.406970 \odot \odot$ & -0.07972000 \\
\hline 0 & -1.68913000 & ๑. 64621600 & -0.92688700 \\
\hline 0 & -2.89592600 & -0.97194600 & -0.05240300 \\
\hline
\end{tabular}

\# b3lyp/tz2p

(1R, 6R)-2a B3LYP/TZ2P Geometry

\section{$\odot 1$}

$\begin{array}{lr}\mathrm{C} & 0.87940800 \\ \mathrm{C} & 2.05377300 \\ \mathrm{C} & 1.63460400 \\ \mathrm{C} & 0.43172700 \\ \mathrm{C} & -0.69051200 \\ \mathrm{H} & -1.20642200 \\ \mathrm{H} & 2.7646540 \odot \\ \mathrm{H} & 1.28794200 \\ \mathrm{H} & 0.36741800 \\ \mathrm{C} & -\odot .12717500 \\ \mathrm{H} & -0.94918900 \\ \mathrm{H} & 0.04830900 \\ \mathrm{H} & 0.84040800 \\ \mathrm{H} & 2.59848900 \\ \mathrm{O} & 2.26023600 \\ \mathrm{C} & 0.22905500 \\ \mathrm{H} & 0.9948080 \odot \\ \mathrm{H} & -0.51103500\end{array}$

$\begin{array}{rr}1.2036700 \odot & 0.9446330 \odot \\ 0.3374360 \odot & 0.4471580 \odot \\ -0.8854920 \odot & -0.3474950 \odot \\ -1.6572810 \odot & 0.1730330 \odot \\ -0.8339220 \odot & 0.8587220 \odot \\ -1.5122820 \odot & 1.5338170 \odot \\ 0.9066580 \odot & -0.1502570 \odot \\ 1.9255850 \odot & 1.6552390 \odot \\ -0.1036190 \odot & 2.5708980 \odot \\ 0.3272750 \odot & 1.6970390 \odot \\ 0.9357620 \odot & 2.0777810 \odot \\ -2.2873190 \odot & -0.6275460 \odot \\ -2.3381970 \odot & 0.9273960 \odot \\ -0.0405110 \odot & 1.3193500 \odot \\ -1.2715070 \odot & -1.3093220 \odot \\ 2.0191500 \odot & -\odot .1830440 \odot \\ 2.6426730 \odot & -\odot .6521300 \odot \\ 2.7018970 \odot & \odot .2400680 \odot\end{array}$

$\begin{array}{ll}1.20367000 & 0.94463300 \\ \odot .33743600 & \odot .44715800\end{array}$

$-0.88549200 \quad-0.34749500$

$-1.65728100 \quad 0.17303300$

- $0.83392200 \quad 0.85872200$

$\odot .90665800$

$1.92558500 \quad 1.65523900$

$-0.10361900 \quad 2.57089800$

$0.32727500 \quad 1.69703900$

$-2.28731900$

$-2.33819700 \quad 0.92739600$

$-0.04051100 \quad 1.31935000$

$-1.27150700 \quad-1.30932200$

$2.64267300 \quad-0.65213000$

๑. 24006800 


\begin{tabular}{|c|c|c|c|}
\hline C & -0.43523500 & 1.22494700 & -1.2986340 \\
\hline $\mathrm{H}$ & -0.71102300 & 1.90123500 & -2.104086 \\
\hline $\mathrm{H}$ & $\odot .2266850 \odot$ & $\odot .4754050 \odot$ & $-1.729627 C$ \\
\hline C & -1.82945200 & -0.42186600 & -0.081790 \\
\hline 0 & -1.69355300 & 0.61259000 & $-0.946914 C$ \\
\hline 0 & $-2.8952070 \odot$ & $-\odot .9774330 \odot$ & $-0.033594 C$ \\
\hline
\end{tabular}

\# b3pw91/tz2p

$(1 \mathrm{R}, 6 \mathrm{R})$-2a B3PW91/TZ2P Geometry

$\odot 1$

\begin{tabular}{|c|c|c|c|}
\hline C & $\odot .86184500$ & 1.20676000 & $\odot .94261700$ \\
\hline C & 2.04226100 & ๑. 35124700 & 0.46149200 \\
\hline C & 1.64141900 & $-\odot .8694520 \odot$ & $-\odot .33606300$ \\
\hline C & $\odot .43475800$ & -1.64058500 & $\odot .16223100$ \\
\hline C & $-\odot .68854800$ & -0.83127500 & 0.84774000 \\
\hline $\mathrm{H}$ & -1.20305200 & -1.51578100 & 1.52036500 \\
\hline $\mathrm{H}$ & 2.76075500 & 0.92363400 & $-\odot .12595400$ \\
\hline $\mathrm{H}$ & 1.25779300 & 1.93801600 & 1.65293900 \\
\hline $\mathrm{H}$ & $\odot .35608300$ & -0.10197300 & 2.56439200 \\
\hline C & -0.13841000 & ๑. 32717600 & 1.68806200 \\
\hline $\mathrm{H}$ & $-\odot .96697700$ & 0.93002800 & 2.06716700 \\
\hline $\mathrm{H}$ & $\odot .0598290 \odot$ & -2.26421800 & $-\odot .6491300 \odot$ \\
\hline $\mathrm{H}$ & $\odot .84001000$ & -2.33233700 & 0.91044800 \\
\hline $\mathrm{H}$ & 2.57798900 & $-0.0272230 \odot$ & 1.34057300 \\
\hline 0 & 2.28785500 & -1.25957800 & -1.28073200 \\
\hline C & $\odot .21662000$ & 2.00220500 & -0.19418100 \\
\hline $\mathrm{H}$ & $\odot .9814890 \odot$ & 2.62843200 & -0.66352100 \\
\hline $\mathrm{H}$ & -0.53452100 & 2.68432800 & 0.21333900 \\
\hline C & $-\odot .42719800$ & 1.18991400 & -1.30227200 \\
\hline $\mathrm{H}$ & $-\odot .68236300$ & 1.85161300 & $-2.1286200 \odot$ \\
\hline $\mathrm{H}$ & $\odot .24395700$ & 0.43134900 & -1.70768400 \\
\hline C & -1.82804100 & -0.42137100 & $-\odot .08438700$ \\
\hline 0 & -1.68589400 & 0.59176300 & -0.96484300 \\
\hline 0 & -2.89936300 & $-\odot .96138400$ & -0.01764400 \\
\hline
\end{tabular}

\# b3lyp/6-31G*

(1R, 6S ) -2b B3LYP/6-31G* Geometry

$\odot 1$

$\begin{array}{lr}\mathrm{C} & 0.72169800 \\ \mathrm{C} & 1.95540700 \\ \mathrm{C} & 1.74095700 \\ \mathrm{C} & 1.00879100 \\ \mathrm{C} & -0.11322100 \\ \mathrm{H} & -0.24948500 \\ \mathrm{H} & 2.29927300 \\ \mathrm{H} & 1.05220500 \\ \mathrm{H} & 1.00566100 \\ \mathrm{C} & 0.22377900 \\ \mathrm{H} & -0.65699700 \\ \mathrm{H} & 0.64138400\end{array}$
1.42586300
0.70930000
$-0.02243400$
$-0.58911700$
$\odot .31643500$
1.20416400
2.04694400
$-0.83266700$
1.23635800
2.52544600
1.76537700
2.28869800
$-\odot .28113000$ 


\begin{tabular}{|c|c|c|c|}
\hline $\mathrm{H}$ & 1.77700900 & -1.94326200 & $\odot .99270700$ \\
\hline $\mathrm{H}$ & 2.78282100 & 0.76762800 & 0.70033000 \\
\hline 0 & 2.17736500 & -0.88797300 & -1.67313700 \\
\hline C & $-\odot .39055400$ & 1.86667300 & -0.25917500 \\
\hline $\mathrm{H}$ & ๑. .02371000 & 2.61656000 & -0.94482100 \\
\hline $\mathrm{H}$ & -1.17811700 & 2.37528800 & 0.312440 \\
\hline C & -1.03446900 & $\odot .7626200 \odot$ & -1.120219 \\
\hline $\mathrm{H}$ & -1.68958400 & 1.22657500 & -1.86118900 \\
\hline $\mathrm{H}$ & $-\odot .27131600$ & $\odot .21408900$ & -1.68506800 \\
\hline C & -1.92611100 & $-0.2367260 \odot$ & $-\odot .395373$ \\
\hline 0 & -3.08950600 & -0.38346200 & -0.684823 \\
\hline 0 & -1.41963800 & $-1.0336860 \odot$ & 0.588985 \\
\hline
\end{tabular}

\# b3lyp/tz2p

(1R, 6S)-2b B3LYP/TZ2P Geometry

$\odot 1$

$\begin{array}{ll}\mathrm{C} & 0.69726700 \\ \mathrm{C} & 1.94046100 \\ \mathrm{C} & 1.75719600\end{array}$

C 1.02643300

C $\quad-0.10818100$

$\mathrm{H} \quad-0.24283000$

$\mathrm{H} \quad 2.28033600$

$\mathrm{H} \quad 1.01206000$

H $\quad 0.97062300$

C $\quad 0.20065400$

$\mathrm{H} \quad-0.68637700$

$\mathrm{H} \quad 0.68097100$

$\mathrm{H} \quad 1.79096500$

$\mathrm{H} \quad 2.75943500$

$0 \quad 2.21918000$

C $\quad-0.40750500$

$\mathrm{H} \quad 0.00572200$

$\mathrm{H} \quad-1.19739800$

C $\quad-1.03767000$

$\mathrm{H}-1.68291100$

$\mathrm{H} \quad-0.27257500$

C $\quad-1.92739600$

$0 \quad-3.09032800$

$0 \quad-1.41204700$
1.40861200

0.86416200

$-0.51951900$

$-1.53120400$

$-0.99135300$

$-1.69308500$

1.53567900

2. 29778400

0.23478800

0.38006300

0.74577000

$-2.35750100$

$-1.93869900$

0.77694700

$-0.82712200$

1.86218900

2. 62193100

2. 35394900

0.77276000

1. 24408800

0.23496000

$-0.23807900$

$-0.36271800$

$-1.07296000$ $\odot .74188300$

0.00792500

$-0.58744400$

$\odot .28012800$

1.16735500

1.98754800

$-0.77932100$

1. 29238100

2. 52623800

1. 76382800

2.28306200

$-0.33782400$

$\odot .95103100$

0.73077000

$-1.66270700$

$-0.22282900$

$-0.88875100$

$\odot .34921700$

$-1.10575300$

$-1.84263900$

$-1.66764900$

$-0.40496900$

$-0.68100800$

0.53811400

\# b3pw91/tz2p

(1R, 6S)-2b B3PW91/TZ2P Geometry

0
$\mathrm{C}$
$\mathrm{C}$
$\mathrm{C}$
$\mathrm{C}$
$\mathrm{C}$
$\mathrm{H}$
$\mathrm{H}$
0.69609800

1.93527500

1.75028300

1. 00281400

$-0.12334000$

$-0.25461500$

2. 28627100
1.41149100

0.85626500

$-0.52719200$

$-1.52176900$

$-0.97079300$

$-1.66412100$

1. 51898600
0.72677800

$\odot .00818400$

$-0.57411600$

๑. 29031900

1.17255200

2. 00287400

$-0.78327100$ 


\begin{tabular}{|c|c|c|c|}
\hline $\mathrm{H}$ & 1.01006500 & 2.30816700 & 1.26826800 \\
\hline $\mathrm{H}$ & 0.96217800 & 0.25724400 & 2.51968700 \\
\hline C & $\odot .1934350 \odot$ & $\odot .40 \odot \odot 350 \odot$ & 1.7539366 \\
\hline $\mathrm{H}$ & -0.69273500 & ๑ . 77601900 & 2.269844 \\
\hline $\mathrm{H}$ & $\odot .6514580 \odot$ & -2.34830100 & -0.326172 \\
\hline $\mathrm{H}$ & 1.76320200 & -1.93737900 & $\odot .962765$ \\
\hline $\mathrm{H}$ & 2.75103300 & 0.76878500 & 0.736492 \\
\hline 0 & 2.23050300 & $-\odot .85100900$ & -1.635201 \\
\hline C & $-\odot .3967620 \odot$ & $1.8500880 \odot$ & -0.249272 \\
\hline $\mathrm{H}$ & 0.02297100 & 2.59926000 & $-\odot .925038$ \\
\hline $\mathrm{H}$ & -1.19239900 & 2.35285700 & $\odot .307209$ \\
\hline C & -1.01397400 & 0.74723400 & -1.113889 \\
\hline $\mathrm{H}$ & -1.64477400 & 1.20177200 & -1.875319 \\
\hline $\mathrm{H}$ & $-\odot .24101300$ & 0.19162300 & -1.650399 \\
\hline C & -1.91866000 & -0.23711600 & -0.405736 \\
\hline 0 & -3.07891400 & -0.35527800 & -0.6907380 \\
\hline 0 & -1.42317200 & -1.05300900 & 0.556005 \\
\hline
\end{tabular}

\# b3lyp/6-31G*

$(1 \mathrm{R}, 6 \mathrm{~S})-2 \mathrm{C}$ B3LYP/6-31G* Geometry

$\odot 1$

\begin{tabular}{|c|c|}
\hline $\mathrm{H}$ & -1.98664500 \\
\hline C & -1.15436000 \\
\hline C & $-\odot .90815700$ \\
\hline C & 0.41849700 \\
\hline C & -0.18102400 \\
\hline C & $-\odot .65707200$ \\
\hline C & -1.71403700 \\
\hline $\mathrm{H}$ & $-\odot .1823400 \odot$ \\
\hline C & 1.73080100 \\
\hline $\mathrm{H}$ & $-\odot .9017040 \odot$ \\
\hline $\mathrm{H}$ & -1.50483000 \\
\hline C & 1.76434300 \\
\hline C & $-\odot .0766620 \odot$ \\
\hline $\mathrm{H}$ & $-1.5846020 \odot$ \\
\hline $\mathrm{H}$ & 0.69459100 \\
\hline $\mathrm{H}$ & $\odot .5955290 \odot$ \\
\hline $\mathrm{H}$ & $-\odot .2725660 \odot$ \\
\hline 0 & -2.74380800 \\
\hline $\mathrm{H}$ & $\odot .4473590 \odot$ \\
\hline 0 & $\odot .87526400$ \\
\hline $\mathrm{H}$ & $-\odot .55450600$ \\
\hline 0 & 2.54194400 \\
\hline $\mathrm{H}$ & 1.97507500 \\
\hline $\mathrm{H}$ & 2.55346900 \\
\hline
\end{tabular}

$-0.83184200$

$-0.45383500$

0.62615400

1. 51927000

1.82094300

0.88167500

$-0.25670400$

$-0.03623100$

$\odot .69194400$

2. 63922000

1.57364200

$-0.52793800$

$-1.55701400$

0.96936900

2. 48109400

2.19510000

0.72086400

$-0.80130500$

$-1.53824300$

$-1.54057300$

$-2.53261200$

$-0.63412500$

0.38094600

1. 31974000
1.56588000

0.96068700

$-1.40613000$

0.64996700

$-0.74207200$

1.53869100

$-0.45836900$

$-1.89542700$

0.62222800

$-0.61631500$

1.63145300

$-0.28722700$

$\odot .99538800$

$-2.19504200$

1.10090000

$-1.41975900$

2.55521200

$-0.80441000$

1.96041500

$-0.08186300$

0.88696200

$-1.20541300$

1.64808700

๑. 27215100

\# b3lyp/tz2p

(1R,6S)-2c B3LYP/TZ2P Geometry

○ 1

$\mathrm{H}$

$-1.96650700$

$-0.87887100$

1.54608800 


\begin{tabular}{|c|c|c|c|}
\hline c & -1.14563900 & $-0.4822880 \odot$ & ๑.9470990๑ \\
\hline C & $-\odot .9224870 \odot$ & $\odot .6336050 \odot$ & -1.39404600 \\
\hline C & $\odot .3972790 \odot$ & 1.50831600 & $\odot .6651180 \odot$ \\
\hline c & -0.20648300 & 1.82334600 & $-0.7172840 \odot$ \\
\hline C & $-\odot .6655140 \odot$ & 0.84694800 & 1.54377900 \\
\hline C & -1.71571500 & $-\odot .2640490 \odot$ & $-\odot .4595660 \odot$ \\
\hline $\mathrm{H}$ & -0.19545900 & -0.01835100 & -1.88404300 \\
\hline C & 1.71847100 & $\odot .7029800 \odot$ & $\odot .62375100$ \\
\hline $\mathrm{H}$ & $-\odot .93089900$ & 2.62858400 & $-\odot .58018100$ \\
\hline $\mathrm{H}$ & -1.51745900 & 1.52307700 & 1.64716000 \\
\hline C & 1.77671600 & $-\odot .5050850 \odot$ & $-\odot .29309300$ \\
\hline C & $-\odot .0597260 \odot$ & $-1.5716960 \odot$ & $\odot .9650690 \odot$ \\
\hline $\mathrm{H}$ & -1.59692600 & $\odot .97768100$ & -2.17630200 \\
\hline $\mathrm{H}$ & $\odot .6606900 \odot$ & 2.46203300 & 1.12668600 \\
\hline $\mathrm{H}$ & $\odot .5609740 \odot$ & 2.21058600 & -1.38857700 \\
\hline $\mathrm{H}$ & $-\odot .2797520 \odot$ & $\odot .6755160 \odot$ & 2.55159500 \\
\hline 0 & -2.74690200 & -0.79525400 & -0.80144200 \\
\hline $\mathrm{H}$ & $\odot .45580100$ & $-1.5646260 \odot$ & 1.92799300 \\
\hline 0 & $\odot .9 \odot 470 \odot \odot \odot$ & $-1.5304090 \odot$ & $-0.1041720 \odot$ \\
\hline $\mathrm{H}$ & $-\odot .5246130 \odot$ & -2.54511400 & $\odot .83641400$ \\
\hline 0 & 2.56459800 & $-\odot .5933510 \odot$ & -1.19628400 \\
\hline $\mathrm{H}$ & 1.96771900 & ๑. 38258800 & 1.63939400 \\
\hline $\mathrm{H}$ & 2.52584800 & 1.34653200 & $\odot .28399400$ \\
\hline
\end{tabular}

\# b3pw91/tz2p

(1R, 6S)-2c B3PW91/TZ2P Geometry

01

\begin{tabular}{|c|c|}
\hline $\mathrm{H}$ & -1.96420200 \\
\hline C & -1.14098300 \\
\hline C & $-\odot .90637800$ \\
\hline C & $\odot .39538400$ \\
\hline C & -0.19954200 \\
\hline C & $-0.6692600 \odot$ \\
\hline C & -1.70501700 \\
\hline $\mathrm{H}$ & -0.17380700 \\
\hline C & 1.70703900 \\
\hline $\mathrm{H}$ & $-\odot .9268900 \odot$ \\
\hline $\mathrm{H}$ & -1.52309100 \\
\hline C & 1.76280800 \\
\hline C & $-\odot .0542 \odot 60 \odot$ \\
\hline $\mathrm{H}$ & -1.57266800 \\
\hline $\mathrm{H}$ & $\odot .66061000$ \\
\hline $\mathrm{H}$ & $\odot .5709150 \odot$ \\
\hline $\mathrm{H}$ & $-\odot .2896070 \odot$ \\
\hline 0 & -2.73630500 \\
\hline $\mathrm{H}$ & $\odot .4640460 \odot$ \\
\hline 0 & $\odot .8990150 \odot$ \\
\hline $\mathrm{H}$ & $-\odot .5188840 \odot$ \\
\hline 0 & 2.54528500 \\
\hline $\mathrm{H}$ & 1.94806300 \\
\hline $\mathrm{H}$ & $2.5224900 \odot$ \\
\hline
\end{tabular}

$-0.87107800$

$-0.47451800$

0.62032600

1.50577600

1. 81162400

0.85514300

$-0.26513600$

$-0.03865400$

0.69641700

2. 61731700

1.53241800

$-0.50547500$

$-1.55607800$

0.95406700

2. 46260300

2. 19743200

0.69171900

$-0.79320400$

$-1.53278600$

$-1.52952500$

$-2.53393400$

$-0.59163700$

0.37238900

1.33496000
1.54296800

0.94491700

$-1.39208500$

$\odot .65822000$

$-0.72319800$

1.53267200

$-0.45979200$

$-1.86765100$

0.62636800

$-0.59442000$

1.62537600

$-0.29005900$

$\odot .97269600$

$-2.18729800$

1.11558100

$-1.39387700$

2.54559700

$-0.80113100$

1.93599800

$-0.09492800$

$\odot .86462900$

$-1.19668800$

1.64424200

$\odot .29239000$

\# b3lyp/6-31G* 
(1S, 6R)-2d B3LYP/6-31G* Geometry

\begin{tabular}{|c|c|c|c|}
\hline & & & \\
\hline $\mathrm{H}$ & -1.31672000 & -0.95816800 & 1.94743000 \\
\hline C & $-\odot .75342800$ & $-\odot .46618200$ & 1.14785900 \\
\hline C & -1.16586500 & ๑. $\odot 88130 \odot \odot$ & -1.37046400 \\
\hline C & $-\odot .05701300$ & 1.80627600 & $\odot .2416940 \odot$ \\
\hline C & $-\odot .8543090 \odot$ & 1.57315000 & -1.06140800 \\
\hline C & -0.72125600 & 1.05104300 & 1.40102900 \\
\hline C & -1.52334000 & -0.75666100 & -0.14942500 \\
\hline $\mathrm{H}$ & $-\odot .2981240 \odot$ & $-\odot .3846770 \odot$ & -1.84755000 \\
\hline C & 1.44549900 & $1.5068240 \odot$ & $\odot .12484800$ \\
\hline $\mathrm{H}$ & -1.80529300 & 2.11187000 & -0.96173700 \\
\hline $\mathrm{H}$ & -1.74860900 & 1.41708600 & 1.52969100 \\
\hline C & 1.51588700 & $-\odot .94872700$ & $-\odot .0987740 \odot$ \\
\hline C & $\odot .66529000$ & -1.10365800 & 1.15728400 \\
\hline $\mathrm{H}$ & -1.99382900 & $\odot .0 \odot \odot 3780 \odot$ & -2.08035700 \\
\hline $\mathrm{H}$ & -0.10483900 & 2.88049700 & 0.47177200 \\
\hline $\mathrm{H}$ & $-\odot .33187400$ & $2.0216120 \odot$ & -1.91476000 \\
\hline $\mathrm{H}$ & $-\odot .1968580 \odot$ & 1.25573200 & 2.34449600 \\
\hline 0 & -2.37919300 & -1.61847800 & -0.19171400 \\
\hline $\mathrm{H}$ & 1.21723800 & $-\odot .69907300$ & $2.0176900 \odot$ \\
\hline 0 & 1.80293000 & $\odot .2967300 \odot$ & $-\odot .5668000 \odot$ \\
\hline $\mathrm{H}$ & $\odot .57843000$ & -2.18196900 & 1.30311900 \\
\hline 0 & 1.93237400 & -1.89868500 & $-\odot .71708000$ \\
\hline $\mathrm{H}$ & 1.91582800 & 1.51834000 & 1.11759200 \\
\hline $\mathrm{H}$ & 1.92696200 & 2.28066300 & $-\odot .47848300$ \\
\hline
\end{tabular}

\# b3lyp/tz2p

(1S, 6R)-2d B3LYP/TZ2P Geometry

$\odot 1$

\begin{tabular}{|c|c|c|c|}
\hline $\mathrm{H}$ & -1.30956100 & -0.96657400 & 1.93423600 \\
\hline C & -0.75337400 & -0.47314200 & 1.13824600 \\
\hline C & -1.16573300 & ๑. . 08175100 & -1.36837800 \\
\hline C & -0.06333400 & 1.79628700 & 0.24243600 \\
\hline C & $-\odot .85136300$ & 1.56323100 & -1.06146900 \\
\hline C & -0.72660400 & 1.03966600 & 1.39540600 \\
\hline C & -1.52811500 & $-\odot .75564800$ & -0.15188700 \\
\hline $\mathrm{H}$ & $-\odot .2999030 \odot$ & $-\odot .39442200$ & -1.83356900 \\
\hline C & 1.43807700 & 1.51109100 & 0.13564300 \\
\hline $\mathrm{H}$ & -1.79608600 & 2.10274400 & -0.97131300 \\
\hline $\mathrm{H}$ & -1.75033300 & 1.39957500 & 1.5219810 \\
\hline C & 1.52328200 & -0.94305600 & -0.10078300 \\
\hline C & $\odot .66240200$ & -1.10753800 & 1.14188800 \\
\hline $\mathrm{H}$ & $-1.9844500 \odot$ & -0.00432200 & -2.08031000 \\
\hline $\mathrm{H}$ & -0.11739500 & 2.86471800 & $\odot .4712240$ \\
\hline $\mathrm{H}$ & -0.32316900 & $2.0032500 \odot$ & -1.90823500 \\
\hline $\mathrm{H}$ & -0.20751200 & 1.24129100 & 2.3354700 \\
\hline 0 & -2.39514600 & -1.59792800 & $-\odot .1883370 \odot$ \\
\hline $\mathrm{H}$ & $1.2099000 \odot$ & $-\odot .7098250 \odot$ & 2.00103500 \\
\hline 0 & 1.81689400 & $\odot .3021740 \odot$ & $-0.553737 \odot$ \\
\hline $\mathrm{H}$ & $\odot .57549500$ & -2.18127000 & 1.2809820 \\
\hline 0 & 1.95049100 & -1.88431300 & -0.7130996 \\
\hline $\mathrm{H}$ & 1.89986700 & 1.52466300 & 1.125653 \\
\hline
\end{tabular}




\begin{tabular}{|c|c|c|c|}
\hline $\mathrm{H}$ & 1.91380600 & 2.28484700 & -0.46238100 \\
\hline \multicolumn{4}{|c|}{ \# b3pw91/tz2p } \\
\hline & ГZ2P Geometry & & \\
\hline & & & \\
\hline H & $-1.3299030 \odot$ & $-\odot .9273440 \odot$ & $1.9361240 \odot$ \\
\hline C & $-\odot .7624940 \odot$ & $-\odot .44775400$ & 1.13766900 \\
\hline C & -1.14513600 & ๑. . 08693100 & -1.36606700 \\
\hline C & $-\odot . \odot 287460 \odot$ & 1.78980600 & $\odot .2291220 \odot$ \\
\hline C & $-\odot .8080810 \odot$ & 1.55968000 & -1.07339400 \\
\hline C & $-\odot .7148540 \odot$ & 1.06133400 & 1.37940600 \\
\hline C & -1.53443800 & $-\odot .7267940 \odot$ & $-\odot .1488390 \odot$ \\
\hline H & $-\odot .28243700$ & -0.41255000 & -1.81600900 \\
\hline C & 1.46288500 & 1.47222400 & $\odot .13814600$ \\
\hline H & $-1.7450010 \odot$ & 2.11690700 & $-\odot .9945260 \odot$ \\
\hline $\mathrm{H}$ & -1.73466800 & 1.44011600 & 1.49229400 \\
\hline C & $1.4945960 \odot$ & $-\odot .9638990 \odot$ & $-0.0943710 \odot$ \\
\hline C & 0.63893900 & -1.09715000 & 1.14924300 \\
\hline H & -1.95701100 & $\odot .00544000$ & -2.08808300 \\
\hline $\mathrm{H}$ & $-\odot .0625690 \odot$ & $2.8625800 \odot$ & $\odot .4469340 \odot$ \\
\hline $\mathrm{H}$ & $-\odot .2672180 \odot$ & 1.98386700 & -1.92181200 \\
\hline H & $-\odot .2011150 \odot$ & 1.26433400 & 2.32369500 \\
\hline 0 & -2.41949100 & $-1.5480490 \odot$ & $-\odot .1814590 \odot$ \\
\hline $\mathrm{H}$ & 1.19455800 & -0.69264600 & 2.00166500 \\
\hline 0 & 1.82068600 & $\odot .26677400$ & $-0.5492180 \odot$ \\
\hline H & $\odot .540 \odot \odot 7 \odot \odot$ & -2.16886400 & 1. $3050320 \odot$ \\
\hline 0 & 1.89301400 & -1.91794500 & $-\odot .7040460 \odot$ \\
\hline H & 1.91320100 & 1.47373000 & 1.13553500 \\
\hline H & $1.9624700 \odot$ & 2.24192700 & $-\odot .4485690 \odot$ \\
\hline
\end{tabular}

